\title{
Identification of key genes and long non-coding RNA associated ceRNA networks in hepatocellular carcinoma
}

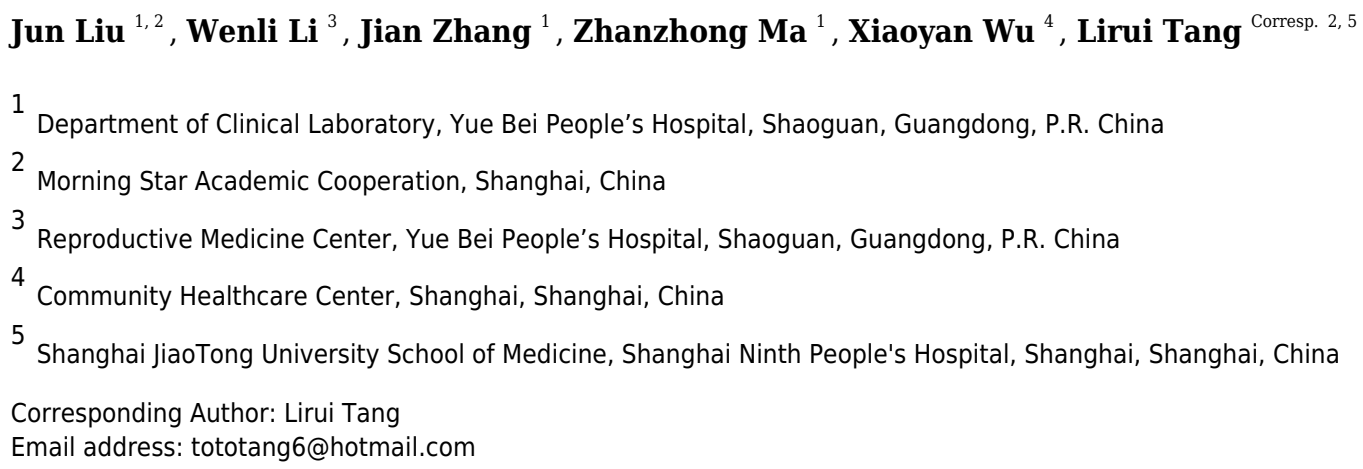

Background. Hepatocellular carcinoma (HCC) is one of the leading causes of cancerrelated deaths worldwide. Although multiple efforts have been made to understand the development of HCC, morbidity and mortality rates remain high. In this study, we aimed to discover the mRNAs and long non-coding RNAs (IncRNAs) that contribute to the progression of HCC. We constructed a IncRNA-related competitive endogenous RNA (ceRNA) network to elucidate the molecular regulatory mechanism underlying HCC. Methods. A microarray dataset (GSE54238) containing information about both mRNAs and IncRNAs was downloaded from the GEO database. Differentially expressed genes (DEGs) and IncRNAs (DEIncRNAs) in tumor tissues and non- cancerous tissues were identified using the limma package of the $\mathrm{R}$ software. The miRNAs that are targeted by DElncRNAs were predicted using miRcode, while the target mRNAs of miRNAs were retrieved from miRDB, miRTarBas, and TargetScan. Functional annotation and pathway enrichment of DEGs were performed using the EnrichNet website. We constructed a protein-protein interaction (PPI) network of DEGs using STRING, and identified the hub genes using Cytoscape. Survival analysis of the hub genes and DEIncRNAs was performed using the GEPIA database. The expression of molecules with prognostic values was validated on the UALCAN database. The hepatic expression of hub genes was examined using The Human Protein Atlas. The hub genes and DEIncRNAs with prognostic values as well as the predictive miRNAs were selected to construct the ceRNA networks. Results. We found that 10 hub genes (KPNA2, MCM7, CKS2, KIF23, HMGB2, ZWINT, E2F1, MCM4, H2AFX, and EZH2) and four IncRNAs (FAM182B, SNHG6, SNHG1, and SNHG3) with prognostic values were overexpressed in the hepatic tumor samples. We also constructed a network containing 10 IncRNA-miRNA-mRNA pathways, which might be responsible for 
regulating the biological mechanisms underlying HCC. Conclusion. We found that the 10 significantly overexpressed hub genes and four IncRNAs were negatively correlated with the prognosis of HCC. Further, we suggest that InCRNA SNHGI and the SNHG3-related ceRNAs can be potential research targets for exploring the molecular mechanisms of HCC. 
1 Identification of key genes and long non-coding RNA2 associated ceRNA networks in hepatocellular

3 carcinoma

4

5

${ }^{1}$ Department of Clinical Laboratory, Yue Bei People's Hospital, Shaoguan, Guangdong, People's Republic of China

${ }^{2}$ Reproductive Medicine Center, Yue Bei People's Hospital, Shaoguan, Guangdong, People's Republic of China ${ }^{3}$ Shanghai Ninth People's Hospital, Shanghai JiaoTong University School of Medicine, Shanghai, People's Republic of China

${ }^{4}$ Community Healthcare Center in Liangcheng District, Shanghai, People's Republic of China ${ }^{5}$ Morning Star Academic Cooperation, Shanghai, People's Republic of China

Corresponding Author:

Lirui Tang

No. 639, Zhizaoju Road, Shanghai 200011, People's Republic of China

Email address: tototang6@hotmail.com 


\section{Abstract}

43 Background. Hepatocellular carcinoma (HCC) is one of the leading causes of cancer-related

44

45

46

47

48

49

50

51

52

53

54

55

56

57

58

59

60

61

62

63

64

65

66

67

68

69

70

\section{1}

72

73

74

75

76

77

78

79

80

81

deaths worldwide. Although multiple efforts have been made to understand the development of HCC, morbidity and mortality rates remain high. In this study, we aimed to discover the mRNAs and long non-coding RNAs (lncRNAs) that contribute to the progression of HCC. We constructed a lncRNA-related competitive endogenous RNA (ceRNA) network to elucidate the molecular regulatory mechanism underlying HCC.

Methods. A microarray dataset (GSE54238) containing information about both mRNAs and lncRNAs was downloaded from the GEO database. Differentially expressed genes (DEGs) and lncRNAs (DElncRNAs) in tumor tissues and non-cancerous tissues were identified using the limma package of the R software. The miRNAs that are targeted by DElncRNAs were predicted using miRcode, while the target mRNAs of miRNAs were retrieved from miRDB, miRTarBas, and TargetScan. Functional annotation and pathway enrichment of DEGs were performed using the EnrichNet website. We constructed a protein-protein interaction (PPI) network of DEGs using STRING, and identified the hub genes using Cytoscape. Survival analysis of the hub genes and DElncRNAs was performed using the GEPIA database. The expression of molecules with prognostic values was validated on the UALCAN database. The hepatic expression of hub genes was examined using The Human Protein Atlas. The hub genes and DElncRNAs with prognostic values as well as the predictive miRNAs were selected to construct the ceRNA networks.

Results. We found that 10 hub genes (KPNA2, MCM7, CKS2, KIF23, HMGB2, ZWINT, E2F1, MCM4, H2AFX, and EZH2) and four lncRNAs (FAM182B, SNHG6, SNHG1, and SNHG3) with prognostic values were overexpressed in the hepatic tumor samples. We also constructed a network containing 10 lncRNA-miRNA-mRNA pathways, which might be responsible for regulating the biological mechanisms underlying HCC.

Conclusion. We found that the 10 significantly overexpressed hub genes and four lncRNAs were negatively correlated with the prognosis of HCC. Further, we suggest that lncRNA SNHG1 and the SNHG3-related ceRNAs can be potential research targets for exploring the molecular mechanisms of HCC.

\section{Introduction}

Hepatocellular carcinoma (HCC) is the third-largest cause of cancer-related deaths worldwide. There has been an upward trend in the incidence of HCC over recent decades. Importantly, the incidence and mortality of HCC have increased in certain non-traditional high-risk regions such as North America and several European regions. In the United States, HCC has already become the fastest-rising contributor for cancer-related deaths.(Kulik \& El-Serag 2019) Several primary diseases, including chronic hepatitis $\mathrm{C}$ virus (HCV), hepatitis B virus (HBV), heavy alcohol consumption, and diabetes, have been confirmed as the main risk factors for HCC.(Singal \& ElSerag 2015) Due to the high morbidity and mortality as well as the diversity of risk factors associated with HCC, there has been great emphasis on exploring early detection markers, novel therapeutic targets, and prognostic biomarkers. Nevertheless, factors such as high rates of

Peer) reviewing PDF | (2019:07:39075:2:0:NEW 5 Oct 2019) 
82 recurrence and metastasis, complexity in treatment and the subsequent rise in financial burden

83 has been a challenge for practitioners globally. Fortunately, the discovery of a variety of non-

84 coding RNAs (ncRNAs), such as lncRNA and miRNA, has facilitated the medical researchers to

85 comprehensively understand HCC.

86 lncRNAs belong to a class of RNAs that do not encode for proteins. These RNAs are more than

87200 nucleotides in length, and are involved in regulating various cellular processes, such as

88 chromatin remodeling, epigenetic modifications, and protein expression. Dysregulation of

89 lncRNAs has been found to promote tumorigenesis and metastasis and is linked with poor

90 prognosis in HCC.(Abbastabar et al. 2018)

91 miRNAs are a type of ncRNAs that have been found to be evolutionarily conserved. They are

92 endogenous and have an average length of 22-nt. miRNAs can fine-tune cellular processes in

93 response to physiological and pathological changes by mediating post-transcriptional regulation

94 of the target genes. The dysregulation of miRNAs and the subsequent anomalous interactions

95 with other molecules have profound implications on the initiation, development, and therapy of

96 HCC.(Borel et al. 2012)

97 A growing body of evidence has shown that lncRNAs and miRNAs not only affect tumor

98 progression separately but also cooperate with each other in modulating the cancer-related genes

99 or pathways. Specifically, IncRNAs bind to mRNAs preventing their interaction with miRNAs and

100 subsequently inhibit the miRNAs. On the other hand, miRNAs repress their target genes when

101 aberrantly expressed. Therefore, the interaction between three types of RNAs results in the

102 formation of a lncRNA-miRNA-mRNA network, which gives rise to competing endogenous

103 RNA (ceRNA). The crosstalk mediated by ceRNA assists in coordinating a large number of

104 biological processes. However, in pathological conditions, this can lead to the development of

105 tumorigenesis.(Karreth \& Pandolfi 2013)

106 In this study, we aimed at exploring the functional significance of lncRNA-related ceRNA

107 networks in HCC based on the ceRNA network theory. We analyzed a microarray dataset

108 containing information about both lncRNA and mRNA obtained from hepatic cancer tissues as

109 well as non-tumor tissues to obtain the DElncRNAs and DEGs. After the target miRNAs of the

110 DElncRNAs as well as the potential upregulated mRNAs of miRNAs were predicted using

111 online tools, the mRNAs that overlapped with the DEGs were selected for further analysis. The

112 DEGs were used to construct a PPI network complex, and we used this information to identify

11310 hub genes. Further, we performed survival analysis on all the hub genes and DElncRNAs.

114 Eventually, 10 hub genes and four DElncRNAs with prognostic values were found to be

115 upregulated in HCC. These hub genes and DElncRNAs as well as the predictive miRNAs were

116 used to construct the ceRNA network.

117

118 Materials \& Methods

119 Data acquisition and preprocessing

120 The GSE54238 expression profile was obtained from the Gene Expression Omnibus (GEO,

121 https://www.ncbi.nlm.nih.gov/geo/), which was imputed on GPL16955 (Arraystar human

Peer] reviewing PDF | (2019:07:39075:2:0:NEW 5 Oct 2019) 
122 lncRNA microarray V1-100309) and was found to contain 13 advanced HCC and 10 normal

123 sample tissues. The downloaded raw data were preprocessed, including background adjustment,

124 normalization, and gene biotype re-annotation.

125

\section{Acquisition and analysis of expression profiles}

The DElncRNAs and DEGs in the HCC and normal tissues were calculated using the limma package of R software (https://www.r-project.org/). mRNAs with a $\mid \log$ fold change (logFC) $\mid \geq 1$ and an adjusted P-value $<0.05$ were considered as the selection criteria of DEGs for subsequent analysis. The heat maps of lncRNAs and DEGs were drawn using the pheatmap package of the R software.

132

\section{Construction of the ceRNA network}

134

The interaction of miRNAs with DElncRNAs was predicted based on the file "Highly conserved

135 microRNA families", which was downloaded from a highly reliable online miRNA reference database, miRcode (http://www.mircode.org/).(Jeggari et al. 2012) The prediction of target mRNAs of miRNAs was performed using three databases, miRDB (http://www.mirdb.org/), (Wong \& Wang 2015) miRTarBas (http://mirtarbase.mbc.nctu.edu.tw), (Hsu et al. 2014) and TargetScan (http://www.targetscan.org) (Park \& Kim 2013). We only selected the mRNAs that overlapped with DEGs for constructing the lncRNA-miRNA-mRNA network. The ceRNA network was visualized by Cytoscape (https://www.cytoscape.org/).(Shannon et al. 2003)

142

\section{Functional annotation and pathway enrichment analysis of DEGs}

144 DEGs were analyzed by Gene Ontology (GO) and Kyoto Encyclopedia of Genes and Genomes

145 (KEGG) analysis on Enrich (http://www.enrichnet.org/) website to perform functional annotation and pathway enrichment.

\section{Construction of the PPI network and screening for the hub genes}

The protein-protein interaction (PPI) network of the targeted DEGs was constructed using STRING (https://string-db.org/) and the Cytoscape. The non-interacting genes were excluded in order to simplify the PPI network. The top 10 genes with the highest degree of connection to the others were considered as hub genes based on the analysis using CytoHubba from Cytoscape.

\section{Reconstruction of the ceRNA network}

156

The hub genes and DElncRNAs were retrieved using the median value as the cut-off on the Gene Expression Profiling Interactive Analysis (GEPIA, http://gepia.cancer-pku.cn/) website, which is based on TCGA. Survival analyses were performed on these newly screened hub genes as well as the lncRNAs to obtain their prognostic values. Then, the hub genes and lncRNAs that showed statistical differences in the survival analysis were used to reconstruct a new ceRNA network. The expression of lncRNAs and hub genes with the most prominent statistical differences in survival was tested and verified using the UALCAN database (http://ualcan.path.uab.edu/). The 
162 hepatic expression of hub genes was examined using the Human Protein Atlas database

163 (https://www.proteinatlas.org/). Correlation analyses were conducted to analyze the correlations

164 between the expression of each lncRNA and its corresponding hub genes in the ceRNA network

165 on GEPIA.

166

167

168

169

170

171

172

173

174

175

176

177

178

179

180

181

182

183

184

185

186

187

188

189

190

191

192

193

194

195

196

197

198

199

\section{Results}

\section{Identification of DEIncRNAs and DEGs}

In total, 10 tissue samples from the control and 13 from the HCC tissues were available in the GSE54238 dataset. The expression profiles of the mRNAs and lncRNAs were calculated. According to the results analyzed by the limma package, 1673 mRNAs (Fig. 1A) and 12 $\operatorname{lncRNAs}$ (Fig. 1B) were differentially expressed $(\mid \log F C) \mid \geq 1$ and adjusted $\mathrm{P}>0.05)$ and they were defined as DEGs and DElncRNAs, respectively. Out of these, 768 mRNAs and 12 lncRNAs were over-expressed while 904 mRNAs and one lncRNA was downregulated.

\section{Construction of the IncRNA-associated ceRNA network}

Based on the results of the online prediction, we found that the miRNAs might interact with DElncRNAs (Table S1). The target mRNAs of the miRNAs are indicated with blue nodes (Fig. 2). Among all the predictive mRNAs, only the 126 mRNAs that also existed in the DEGs were selected to construct the first ceRNA network. Table S2 shows the details of the 126 mRNAs. The red nodes are DElncRNAs (GAS5, FAM41C, FAM182B, FAM138 B, FAM138C, FAM138D, FAM138E, UCA1, SNHG1, SNHG3, and SNHG6) and the green nodes represent the latently interactive miRNAs.

\section{Functional annotation and pathway enrichment analysis of DEGs}

In order to thoroughly understand the properties and functions of the 126 DEGs in HCC development and progression, we applied functional annotation and pathway enrichment analysis on each of the DEGs from the ceRNA network. The GO analysis indicated that: 1) for biological processes (BP), the DEGs from the ceRNA network were particularly enriched in positive regulation of protein localization on the membrane, positive regulation of protein insertion into mitochondrial membrane involved in apoptotic signaling pathway, positive regulation of mitochondrial outer membrane permeabilization involved in apoptotic signaling pathway, regulation of epithelial cell proliferation, regulation of nuclear-transcribed mRNA catabolic process, deadenylation-dependent decay, positive regulation of epithelial to mesenchymal transition, positive regulation of apoptotic processes, positive regulation of nuclear-transcribed mRNA catabolic process, and positive regulation of gene expression (Fig. 3A); 2) for cellular components $(\mathrm{CC})$, the DEGs were significantly enriched in chromatin, nuclear chromosomes, nuclear chromatin, apical dendrite, cytoplasmic vesicle membrane, $\mathrm{ESC} / \mathrm{E}(\mathrm{Z})$ complex, lipid droplet, chromosome, telomeric region and centrosome (Fig. 3B); 3) for molecular functions 200 (MF), the DEGs were enriched in transcription regulatory region DNA binding, core promoter sequence-specific DNA binding, mRNA 3'-UTR AU-rich region binding, regulatory region 
202 DNA binding, RNA polymerase II activating transcription factor binding, core promoter binding, 203 cadherin binding, L-amino acid transmembrane transporter activity, insulin-like growth factor 204 receptor binding and amino acid transmembrane transporter activity (Fig. 3C). KEGG analysis 205 demonstrated that DEGs were particularly enriched in the cell cycle, microRNAs involved in 206 cancer, central carbon metabolism in cancer, pentose phosphate pathway, PI3K-Akt signaling 207 pathway, fluid shear stress and atherosclerosis, colorectal cancer, non-alcoholic fatty liver 208 disease (NAFLD), small cell lung cancer, and cellular senescence (Fig. 3D).

209

210

211

212

213

214

215

216

217

218

219

220

221

222

223

224

225

226

227

228

229

230

231

232

233

234

235

236

237

238

239

240

241

\section{Construction of the PPI network and identification of the hub genes}

The PPI network complex contained 90 DEGs (Fig. 4A). We evaluating their degree of connection, and identified 10 hub genes (MCM4, CKS2, ZWINT, HMGB2, MCM7, KPNA2, E2F1, H2AFX, KIF23 and EZH2), which were all up-regulated in HCC. The hub genes indicated with red nodes (MCM4, ZWINT, MCM7, and KPNA2) had the strongest connections with others, while those indicated with orange (CKS2, HMGB2, KIF23, E2F1) and yellow nodes (EZH2, H2AFX) had moderate and weak connections, respectively (Fig. 4B).

\section{Survival analysis of hub genes and DEIncRNAs}

We performed survival analysis on the hub genes and DElncRNAs and found that 10 overexpressed hub genes (MCM4, MCM7, ZWINT, KPNA2, CKS2, KIF23, E2F1, HMGB2, $\mathrm{EZH} 2$, and $\mathrm{H} 2 \mathrm{AFX}$ ) were significantly related to poorer prognosis with worse survival times in HCC patients. Among these, KPNA2 and EZH2 had the highest prognostic value (Fig. 5). We used the information from the ICGC database to validate our analyses, and found them to be consistent with our results (Fig. S1). Four DElncRNAs (FAM182B, SNHG1, SNHG3, and SNHG6) were upregulated and were found to be negatively related to the prognosis of $\mathrm{HCC}$, with SNHG3 having the best clinical predictive value (Fig. 6).

\section{Validation of the expression of hub genes and DEIncRNAs}

Expression of the hub genes, as well as the DElncRNAs with prognostic significance, was tested using the UALCAN database in order to verify the differences in expression. Consistent with the results described before, all of the DElncRNAs (Fig. 7) and hub genes (Fig. 8) with prognostic significance were significantly overexpressed in HCC tissues compared with normal ones. Further details of the comparisons are shown in Table S3. In addition, comparative analysis of the hub genes from the ICGC database was consistent with that obtained from the UALCAN database (Fig. S2). Furthermore, hepatic expressions of the hub genes (MCM4, MCM7, ZWINT, KPNA2, CKS2, KIF23, E2F1, HMGB2, EZH2, and H2AFX) were visualized using immunohistochemistry based on The Human Protein Atlas. While MCM4, MCM7, and EZH2 were not detected in non-tumor tissues, they were moderately or highly expressed in tumor tissues. The genes, ZWINT, E2F1, HMGB2, KPNA2, and CKS2, were expressed at low or moderate levels in non-tumor tissues. However, the staining intensity or the range of positive distribution of all the other proteins increased in tumor samples, except for KPNA2 (Fig. 9). 


\section{Reconstruction of the IncRNA-associated ceRNA network}

244

245

246

247

248

249

250

251

252

253

254

255

256

257

258

259

260

261

262

263

264

265

266

267

268

269

270

271

272

273

274

275

276

277

278

279

280

281

The newly screened hub genes and DElncRNAs were used to reconstruct a new ceRNA network. In total, 10 hub genes (KPNA2, MCM7, CKS2, KIF23, HMGB2, ZWINT, E2F1, MCM4, H2AFX, and EZH2), seven miRNAs (hsa-miR-107, hsa-miR-1297, hsa-miR-24-3p, hsa-miR216b-5p, hsa-miR-217, hsa-miR-338-3p, and hsa-miR-107) and four IncRNAs (FAM182B, SNHG6, SNHG1, and SNHG3) were included (Fig. 10). The reconstructed network consisted of 10 lncRNA-miRNA-mRNA pathways, including FAM182B-hsa-miR-107-KIF23, FAM182Bhsa-miR-107-MCM7, SNHG6-hsa-miR-1297-KPNA2, SNHG6-hsa-miR-1297-CKS2, SNHG6hsa-miR-24-3p-H2AFX, SNHG3-hsa-miR-338-3p-ZWINT, SNHG1-hsa-miR-217-EZH2, SNHG1-hsa-miR-23b-3p-HMGB2, SNHG1-hsa-miR-216b-5p-E2F1, and SNHG1-hsa-miR216b-5p-MCM4 (Table 1). lncRNA SNHG1 had the highest number of connections with the hub genes.

\section{Co-expression analysis of IncRNAs and hub genes from the ceRNA network}

Correlation analyses showed that expressions of all the lncRNAs were correlated with that of their corresponding mRNAs (Fig. 11A-F). SNHG1 had the strongest correlations with its hub genes as the correlation coefficient for E2F1, EZH2, HMGB2, and MCM4 being 0.67, 0.77, 0.72, and 0.7 , respectively (Fig. 11C-F). All of these indicate a strong correlation. SNHG3, the lncRNA with the most valuable clinical significance, also showed a strong correlation with ZWINT (R=0.6, Fig. 11G). In terms of expression, FAM182B and SNHG6 were moderately related to their corresponding mRNAs with correlation coefficients ranging from 0.51 to 0.67 .

\section{Discussion}

In this study, 10 hub genes and four lncRNAs were found to be overexpressed in HCC tissues. Meanwhile, survival analysis results confirmed that they were potential candidates for prognostic predictions in HCC patients. Among all the hub genes, KPNA2 and EZH2, which are tumorpromoting genes, have been extensively studied and were found to be the most effective predictors of survival time. SNHG3, a lncRNA that is relatively less known, had the highest predictive value for clinical HCC prognosis.

None of the hub genes were completely new in the field of HCC. It has been shown that HCC patients who had higher expression of KPNA2 in the nucleus have poorer prognosis and higher risk of recurrence.(Jiang et al. 2014b) KPNA2 imparts proliferative and metastatic abilities to the HCC cells by aiding the entry of certain transcriptional factors, such as PLAG2, into the nucleus.(Hu et al. 2014) MCM7 has been identified as a prognostic marker of HCC that promotes cell proliferation and tumorigenicity by suppressing cyclin D1 expression via the activation of the MAPK pathway. Through this mechanism, MCM7 initiates DNA replication, which is a key process in cell cycle progression.(Qu et al. 2017) CKS2 is activated by the Wnt/ $\beta$ catenin signaling pathway and promotes cell proliferation and inhibits apoptosis, which makes 
282 the HCC cells more aggressive as its overexpression is more frequently observed in poorly 283 differentiated tumors.(Li et al. 2018; Shen et al. 2010) KIF23 depletion inhibits tumor formation 284 by inducing apoptosis in certain types of cancer, such as lung adenocarcinomas. However, there 285 are diverse clinical research reports on HCC that contradict our results. According to a previous 286 study, KIF23, which has two splice variants, V1 and V2, is overexpressed in HCC tissues, while 287 it is almost undetectable in normal tissues. Patients with positive V1 expression, which is mainly 288 located inside the nucleus, have better overall five-year survival than those with negative results. 289 However, KIF23 V2 had no correlation with overall survival (OS). Therefore, further in-depth 290 studies should be undertaken to evaluate the function of KIF23 in HCC.(Sun et al. 2015) 291 HMGB2 overexpression was detected at both mRNA and protein levels in tumor tissues from

292

293

294

295

296

297

298

299

300

301

302

303

304

305

306

307

308

309

310

311

312

313

314

315

316

317

318

319

320

321 HCC patients. It is associated with shorter OS, and is considered to be an independent prognostic factor. In an in vitro study, HMGB2 was found to promote HCC cell proliferation and impair drug sensitivity.(Kwon et al. 2010) ZWINT protein is elevated in HCC tissues and shows correlation with tumor size and number. HCC patients with high expression of ZWINT tend to experience higher rates of tumor recurrence. ZWINT may enhance cell proliferation by disturbing the expression of cell cycle-related proteins, such as PCNA and cyclin B1, which results in shorter OS.(Ying et al. 2018) E2F1 protein is activated to a greater extent in tumor tissues compared to that in the normal liver or adjacent non-tumor tissues from the same HCC patients.(Feng et al. 2015) In mice with copy number gains in E2F1, dosage-dependent spontaneous tumors only occurred in the liver, which indicates that E2F1 is specifically involved in the promotion of hepatic tumorigenesis.(Kent et al. 2017) The survival analysis from another dataset, GSE14520, supported our results, which showed that MCM4 enrichment had significant association with the OS in HCC patients in the background of hepatitis B infection.(Liao et al. 2018) H2AFX is upregulated and activated in HCC, as shown by the increase in the levels of total and phosphorylated H2AFX in tumor tissues.(Evert et al. 2013) In addition, H2AFX together with another seven genes, constitutes a prognostic signature for $\mathrm{HCC}$ with $\mathrm{C}$-indexes of $0.776,0.745$, and 0.789 for 1-, 3-, and 5-year OS, respectively.(Zhang et al. 2019b) EZH2 is important in determining the character of hepatic tumors and immunohistochemistry results showed that this protein was positively stained in various types of malignant liver tumors. However, EZH2 has not been detected in benign hepatic diseases, such as adenomas or cirrhotic nodules.(Hajosi-Kalcakosz et al. 2012) By inhibiting the natural killer cell-mediated eradication of tumor cells, EZH2 may create an immune microenvironment that is conducive to HCC cells.(Bugide et al. 2018)

We also constructed a lncRNA-related ceRNA network consisting of 10 possible lncRNAmiRNA-mRNA pathways with DEGs and DElncRNAs, in which the DElncRNAs might compete with the DEGs for binding to their target miRNAs. Through this process, the DElncRNAs can regulate the expression and function of DEGs without directly interacting with them. In all the pathways, lncRNA SNHG1 might potentially regulate most of the hub genes, including EZH2, HMGB2, E2F1 and MCM4. It is worth noting that our results demonstrated a 
322

323

324

325

326

327

328

329

330

331

332

333

334

335

336

337

338

339

340

341

342

343

344

345

346

347

348

349

350

351

352

353

354

355

356

357

358

359

360

361

close correlation between the expression of SHNG1 and the four mRNAs, which strengthens the possibility of mutual regulation between them. Since all the mRNAs and lncRNAs in this network originated from the same dataset, we believed the predictive regulatory relationships are convincing, and are worth investigating further.

SNHG3 was found to be overexpressed in HCC tissues as compared to normal hepatic samples in this study. In addition, lncRNA SNHG3 was the most valuable prognostic factor for HCC. Consistent with our finding, SNHG3 expression has been proven to be negatively associated with the overall survival, recurrence-free survival and disease-free survival in HCC patients. (Zhang et al. 2016c) SNHG3 promotes metastasis and induces sorafenib resistance by inducing EMT via the miR-128/CD151 pathway in HCC cells.(Zhang et al. 2019a) Although there have been no research investigations on the direct interactions between SNHG3 and its targeting miRNAs in HCC, several studies have found that SNHG3 forms a ceRNA network by sponging miRNAs in other cancers.(Chen et al. 2019; Huang et al. 2017; Wang et al. 2019; Zheng et al. 2019) For example, SNHG3 sponged miRNA-151a-3p (Zheng et al. 2019) and miR-196a-5p (Chen et al. 2019 ) in order to promote cell growth, invasion, and migration in osteosarcoma. miR-338-3p is downregulated in HCC and inhibits its progression in various ways including suppressing cell proliferation (Fu et al. 2012), countering Warburg effects (Nie et al. 2015) as well as inhibiting metastasis (Chen et al. 2017; Zhang et al. 2016d). A diverse array of molecules, such as mineralocorticoid receptors (Nie et al. 2015), hepatitis B virus X (Fu et al. 2012) and circular RNAs (circRNA) (Li et al. 2019) have been validated as the direct upstream regulators of miR-338-3p in HCC. A novel mechanism indicating that miR-338-3p expression might be regulated by lncRNA SNHG3 has been identified. The downstream binding targets of miR-338-3p, including N-cadherin (Chen et al. 2017), MACC1 (Zhang et al. 2016d), HIF-1 $\alpha$ (Xu et al. 2014), and CyclinD1 (Fu et al. 2012) have also been well studied in HCC. However, for the first time, this study has predicted ZWINT as a potential target of miR-338-3p. ZWINT is upregulated in HCC samples as well as cell lines, and promotes cell proliferation by affecting the expression of cell-cycle proteins, such as PCNA, cyclin, and CDK1.(Ying et al. 2018) ZWINT has also been identified as a key biomarker in drug-induced liver injury, which indicates its possible involvement in drug metabolism.(Cho et al. 2016) However, a study conducted in post-surgery HCC patients showed that ZWINT expression was decreased in tumor samples.(Yang et al. 2018b) These contradictory results could be due to the different stages of the disease when these studies were carried out, and due to the diverse causes that lead to development of HCC. Several miRNAs, such as miR-127-3p and miR-1, have been found to directly or potentially target ZWINT and promote tumor progression (Jiang et al. 2014a; Xie et al. 2018). Here, we identified ZWINT as a target of miR-338-3p that might form a ceRNA network with lncRNA SNHG3 in HCC.

In this study, we found that lncRNA SNHG1 was negatively associated with HCC prognosis. This result is consistent with previous research, which showed that overexpression of SNHG1 
362

363

364

365

366

367

368

369

370

371

372

373

374

375

376

377

378

379

380

381

382

383

384

385

386

387

388

389

390

391

392

393

394

395

396

397

398

399

400

correlated with larger tumor size, poor differentiation degree, and a worse clinical-stage.(Zhang et al. 2016b) SNHG1 also had the most number of connections with the hub genes, which means that it can most likely regulate the expression of the four mRNAs found in this network through miRNAs. SNHG1 was found to exacerbate HCC by inhibiting miR-195 directly.(Zhang et al. 2016a) However, to our knowledge, this study has reported for the first time that in HCC, hsamiR-217, has-miR-216b-5p, and hsa-miR-23b-3p might be the other targets of SNHG1. In HCC, decreased miR-217 expression is positively associated with vascular invasion, and TNM stage.(Tian et al. 2017) miR-217 partially inhibits hepatic metastasis by binding to the 3'UTR of MTDH mRNA,(Su et al. 2014; Zhang et al. 2017) which encodes a protein that can induce cell growth and inhibit apoptosis.(Li et al. 2015) However, miR-217 was also found to be overexpressed in HCC and is potentially involved in promoting hepatic disease.(Jiang et al. 2017) miR-217 induces cancer stem cell-like phenotypes resulting in the activation of the Wnt pathway in HCC cells.(Jiang et al. 2017) High miR-217 levels leads to fat deposition in hepatocytes.(Yin et al. 2012) These conflicting results may be attributed to the difference in the cell lines used in the experiments and the different stages of the disease when the studies were conducted.

miR-216b-5p is an anti-tumor miRNA found in several cancers.(He et al. 2019; Ren et al. 2019; Sun et al. 2019b; You et al. 2017) It is believed that miR-216b-5p inhibits HCV by suppressing host autophagy (Huang et al. 2018). Paradoxically, miR-216b-5p represses the expression of UDP-glucuronosyltransferase $2 \mathrm{~B}$, a group of enzymes that detoxifies carcinogens by binding to their 3'UTRs.(Dluzen et al. 2016) In addition, few other studies have also explored how miR216b-5p affects HCC progression.

Low hepatic miR-23b-3p expression is considered as a biomarker of HCC tumorigenesis and progression.(He et al. 2018) Meanwhile, serum miR-23b-3p levels help in distinguishing between the HCV patients that are at high risk of progressing to HCC from those with low risk.(Sun et al. 2019a) Mechanically, miR-23b-3p inhibits the epithelial-mesenchymal transition in HCC cells via sponging by lncRNA HOTAIR from ZEB1.(Yang et al. 2018a) Although it has been speculated that miR-23b-3p is also involved in fat metabolism, the conclusions are inconsistent. A study found that in HepG2 cells, miR-23b-3p inhibits the synthesis of ApoA protein, which is a risk factor for thrombotic diseases (Zeng et al. 2018), whereas another study reported that SIRT1, an enzyme that prevents lipid accumulation, was repressed by hsa-miR23b-3p in hepatocytes.(Borji et al. 2019) Among all the mRNAs in the SNHG1-related ceRNA network, EZH2, a widely investigated oncogene, is highly expressed in HCC tissues and contributes to its progression.(Sudo et al. 2005) Similar to the results in this study, EZH2 has been validated as a direct target of miR-217 in gastric cancer cells.(Chen et al. 2015) Nevertheless, a study conducted in colorectal cancer cells has found that EZH2 promotes cell growth by directly binding to lncRNA SNHG1 without requiring mediation of miRNAs.(Xu et al. 2018) This suggests that EZH2 expression might be regulated by SNHG1 through various pathways. 
401 In HCC, the HMGB2 mRNA is overexpressed and serves as an independent prognostic factor for 402 OS.(Kwon et al. 2010) Similar to our predictive analysis, HMGB2 was found to promote gastric 403 cancer cell autophagy and induce multidrug resistance by directly interacting with miR-23b-

$4043 p$. (An et al. 2015)

405 E2F1 is a transcription factor that has been known to promote HCC progression.(Farra et al. 406 2017) Besides the findings in this study, miR-331-3p (Jin et al. 2019) and miR-34a (Han et al. 407 2019) have also been found to regulate E2F1 expression in HCC cell lines. However, precise 408 interactions between E2F1 and miRNAs need further exploration.

409 MCM4 expression is inversely related to the OS in hepatitis B virus-related HCC. (Liao et al. 410 2018) The gene polymorphism of MCM4 also affects the chances of developing HCC as the 411 patients who carry the MCM4 rs2305952 CC are less likely to have the disease.(Nan et al. 2016) The regulatory mechanism of MCM4 in HCC has been barely explored as there is only one previous study reporting MCM4 as a latent target of miR-122-5p.(Wen et al. 2018) The results of

415

In summary, we identified 10 mRNAs and four lncRNAs that may promote HCC progression.

417 We also constructed the lncRNA-miRNA-mRNA ceRNA network in order to understand the latent regulatory mechanism between mRNAs and ncRNAs. The lncRNA SNHG1 and SNHG3related ceRNAs can be selected as novel targets while exploring the molecular mechanism of HCC.

421

422

\section{Conclusions}

423

In this study, we identified 10 significantly overexpressed hub genes (MCM4, CKS2, ZWINT, HMGB2, MCM7, KPNA2, E2F1, H2AFX, KIF23 and EZH2) and four lncRNAs (FAM182B, SNHG1, SNHG3 and SNHG6) and found them to be negatively correlated with HCC prognosis. research targets for investigating the molecular mechanism of HCC.

428

429

430

\section{Acknowledgements}

431

432

433

434

435

436

437

438

439

440

441

442

None.

Abbastabar M, Sarfi M, Golestani A, and Khalili E. 2018. IncRNA involvement in hepatocellular carcinoma metastasis and prognosis. EXCLI J 17:900-913. 10.17179/excli2018-1541

An Y, Zhang Z, Shang Y, Jiang X, Dong J, Yu P, Nie Y, and Zhao Q. 2015. miR-23b-3p regulates the chemoresistance of gastric cancer cells by targeting ATG12 and HMGB2. Cell Death Dis 6:e1766. 10.1038/cddis.2015.123

Borel F, Konstantinova P, and Jansen PL. 2012. Diagnostic and therapeutic potential of miRNA signatures in patients with hepatocellular carcinoma. J Hepatol 56:1371-1383. 10.1016/j.jhep.2011.11.026

Borji M, Nourbakhsh M, Shafiee SM, Owji AA, Abdolvahabi Z, Hesari Z, Ilbeigi D, Seiri P, and Yousefi Z. 2019. Down-Regulation of SIRT1 Expression by mir-23b Contributes to Lipid Accumulation in HepG2 Cells. Biochem Genet. 10.1007/s10528-019-09905-5 
443 Bugide S, Green MR, and Wajapeyee N. 2018. Inhibition of Enhancer of zeste homolog 2

444

445

446

447

448

449

450

451

452

453

454

455

456

457

458

459

460

461

462

463

464

465

466

467

468

469

470

471

472

473

474

475

476

477

478

479

480

481

482

483

484

485

486

487

488

489

490

491

492

493

$(\mathrm{EZH} 2)$ induces natural killer cell-mediated eradication of hepatocellular carcinoma cells. Proc Natl Acad Sci U S A 115:E3509-E3518. 10.1073/pnas.1802691115

Chen DL, Zhang DS, Lu YX, Chen LZ, Zeng ZL, He MM, Wang FH, Li YH, Zhang HZ, Pelicano $H$, Zhang W, and Xu RH. 2015. microRNA-217 inhibits tumor progression and metastasis by downregulating EZH2 and predicts favorable prognosis in gastric cancer. Oncotarget 6:10868-10879. 10.18632/oncotarget.3451

Chen J, Wu Z, and Zhang Y. 2019. LncRNA SNHG3 promotes cell growth by sponging miR196a-5p and indicates the poor survival in osteosarcoma. Int J Immunopathol Pharmacol 33:2058738418820743. 10.1177/2058738418820743

Chen JS, Liang LL, Xu HX, Chen F, Shen SL, Chen W, Chen LZ, Su Q, Zhang LJ, Bi J, Zeng WT, Li W, Ma N, and Huang XH. 2017. miR-338-3p inhibits epithelial-mesenchymal transition and metastasis in hepatocellular carcinoma cells. Oncotarget 8:71418-71429. 10.18632/oncotarget.10138

Cho H, Kim H, Na D, Kim SY, Jo D, and Lee D. 2016. Meta-analysis method for discovering reliable biomarkers by integrating statistical and biological approaches: An application to liver toxicity. Biochem Biophys Res Commun 471:274-281. 10.1016/j.bbrc.2016.01.082

Dluzen DF, Sutliff AK, Chen G, Watson CJ, Ishmael FT, and Lazarus P. 2016. Regulation of UGT2B Expression and Activity by miR-216b-5p in Liver Cancer Cell Lines. J Pharmacol Exp Ther 359:182-193. 10.1124/jpet.116.235044

Evert M, Frau M, Tomasi ML, Latte G, Simile MM, Seddaiu MA, Zimmermann A, Ladu S, Staniscia T, Brozzetti S, Solinas G, Dombrowski F, Feo F, Pascale RM, and Calvisi DF. 2013. Deregulation of DNA-dependent protein kinase catalytic subunit contributes to human hepatocarcinogenesis development and has a putative prognostic value. $\mathrm{Br} \mathrm{J}$ Cancer 109:2654-2664. 10.1038/bjc.2013.606

Farra R, Grassi G, Tonon F, Abrami M, Grassi M, Pozzato G, Fiotti N, Forte G, and Dapas B. 2017. The Role of the Transcription Factor E2F1 in Hepatocellular Carcinoma. Curr Drug Deliv 14:272-281. 10.2174/1567201813666160527141742

Feng H, Yu Z, Tian Y, Lee YY, Li MS, Go MY, Cheung YS, Lai PB, Chan AM, To KF, Chan HL, Sung JJ, and Cheng AS. 2015. A CCRK-EZH2 epigenetic circuitry drives hepatocarcinogenesis and associates with tumor recurrence and poor survival of patients. J Hepatol 62:1100-1111. 10.1016/j.jhep.2014.11.040

Fu X, Tan D, Hou Z, Hu Z, and Liu G. 2012. miR-338-3p is down-regulated by hepatitis B virus $X$ and inhibits cell proliferation by targeting the 3 '-UTR region of CyclinD1. Int J Mol Sci 13:8514-8539. 10.3390/ijms13078514

Hajosi-Kalcakosz S, Dezso K, Bugyik E, Bodor C, Paku S, Pavai Z, Halasz J, Schlachter K, Schaff Z, and Nagy P. 2012. Enhancer of zeste homologue 2 (EZH2) is a reliable immunohistochemical marker to differentiate malignant and benign hepatic tumors. Diagn Pathol 7:86. 10.1186/1746-1596-7-86

Han R, Chen X, Li Y, Zhang S, Li R, and Lu L. 2019. MicroRNA-34a suppresses aggressiveness of hepatocellular carcinoma by modulating E2F1, E2F3, and Caspase-3. Cancer Manag Res 11:2963-2976. 10.2147/CMAR.S202664

He J, Sun M, Geng H, and Tian S. 2019. Long non-coding RNA Linc00518 promotes paclitaxel resistance of the human prostate cancer by sequestering miR-216b-5p. Biol Cell 111:3950. 10.1111/boc.201800054

He RQ, Wu PR, Xiang XL, Yang X, Liang HW, Qiu XH, Yang LH, Peng ZG, and Chen G. 2018. Downregulated miR-23b-3p expression acts as a predictor of hepatocellular carcinoma progression: A study based on public data and RT-qPCR verification. Int $\mathrm{J} \mathrm{Mol} \mathrm{Med}$ 41:2813-2831. 10.3892/ijmm.2018.3513

Hsu SD, Tseng YT, Shrestha S, Lin YL, Khaleel A, Chou CH, Chu CF, Huang HY, Lin CM, Ho SY, Jian TY, Lin FM, Chang TH, Weng SL, Liao KW, Liao IE, Liu CC, and Huang HD.

Peer) reviewing PDF | (2019:07:39075:2:0:NEW 5 Oct 2019) 
494

495

496

497

498

499

500

501

502

503

504

505

506

507

508

509

510

511

512

513

514

515

516

517

518

519

520

521

522

523

524

525

526

527

528

529

530

531

532

533

534

535

536

537

538

539

540

541

542

543

544

2014. miRTarBase update 2014: an information resource for experimentally validated miRNA-target interactions. Nucleic Acids Res 42:D78-85. 10.1093/nar/gkt1266

Hu ZY, Yuan SX, Yang Y, Zhou WP, and Jiang H. 2014. Pleomorphic adenoma gene 1 mediates the role of karyopherin alpha 2 and has prognostic significance in hepatocellular carcinoma. J Exp Clin Cancer Res 33:61. 10.1186/s13046-014-0061-1

Huang H, Zhang C, Wang B, Wang F, Pei B, Cheng C, Yang W, and Zhao Z. 2018.

Transduction with Lentiviral Vectors Altered the Expression Profile of Host MicroRNAs. $J$ Virol 92. 10.1128/JVI.00503-18

Huang W, Tian Y, Dong S, Cha Y, Li J, Guo X, and Yuan X. 2017. The long non-coding RNA SNHG3 functions as a competing endogenous RNA to promote malignant development of colorectal cancer. Oncol Rep 38:1402-1410. 10.3892/or.2017.5837

Jeggari A, Marks DS, and Larsson E. 2012. miRcode: a map of putative microRNA target sites in the long non-coding transcriptome. Bioinformatics 28:2062-2063. 10.1093/bioinformatics/bts344

Jiang C, Yu M, Xie X, Huang G, Peng Y, Ren D, Lin M, Liu B, Liu M, Wang W, and Kuang M. 2017. miR-217 targeting DKK1 promotes cancer stem cell properties via activation of the Wnt signaling pathway in hepatocellular carcinoma. Oncol Rep 38:2351-2359. 10.3892/or.2017.5924

Jiang H, Jin C, Liu J, Hua D, Zhou F, Lou X, Zhao N, Lan Q, Huang Q, Yoon JG, Zheng S, and Lin B. 2014a. Next generation sequencing analysis of miRNAs: MiR-127-3p inhibits glioblastoma proliferation and activates TGF-beta signaling by targeting SKI. Omics 18:196-206. 10.1089/omi.2013.0122

Jiang P, Tang Y, He L, Tang H, Liang M, Mai C, Hu L, and Hong J. 2014b. Aberrant expression of nuclear KPNA2 is correlated with early recurrence and poor prognosis in patients with small hepatocellular carcinoma after hepatectomy. Med Oncol 31:131. 10.1007/s12032014-0131-4

Jin W, Zhong N, Wang L, Yu J, Yin F, and Zhang K. 2019. MiR-331-3p Inhibition of the Hepatocellular Carcinoma (HCC) Bel-7402 Cell Line by Down-Regulation of E2F1. J Nanosci Nanotechnol 19:5476-5482. 10.1166/jnn.2019.16535

Karreth FA, and Pandolfi PP. 2013. ceRNA cross-talk in cancer: when ce-bling rivalries go awry. Cancer Discov 3:1113-1121. 10.1158/2159-8290.CD-13-0202

Kent LN, Bae S, Tsai SY, Tang X, Srivastava A, Koivisto C, Martin CK, Ridolfi E, Miller GC, Zorko SM, Plevris E, Hadjiyannis Y, Perez M, Nolan E, Kladney R, Westendorp B, de Bruin A, Fernandez S, Rosol TJ, Pohar KS, Pipas JM, and Leone G. 2017. Dosagedependent copy number gains in E2f1 and E2f3 drive hepatocellular carcinoma. $J$ Clin Invest 127:830-842. 10.1172/JCI87583

Kulik L, and El-Serag HB. 2019. Epidemiology and Management of Hepatocellular Carcinoma. Gastroenterology 156:477-491 e471. 10.1053/j.gastro.2018.08.065

Kwon JH, Kim J, Park JY, Hong SM, Park CW, Hong SJ, Park SY, Choi YJ, Do IG, Joh JW, Kim DS, and Choi KY. 2010. Overexpression of high-mobility group box 2 is associated with tumor aggressiveness and prognosis of hepatocellular carcinoma. Clin Cancer Res 16:5511-5521. 10.1158/1078-0432.CCR-10-0825

Li Q, Pan X, Zhu D, Deng Z, Jiang R, and Wang X. 2019. Circular RNA MAT2B Promotes Glycolysis and Malignancy of Hepatocellular Carcinoma Through the miR-338-3p/PKM2 Axis Under Hypoxic Stress. Hepatology. 10.1002/hep.30671

Li WF, Ou Q, Dai H, and Liu CA. 2015. Lentiviral-Mediated Short Hairpin RNA Knockdown of MTDH Inhibits Cell Growth and Induces Apoptosis by Regulating the PTEN/AKT Pathway in Hepatocellular Carcinoma. Int J Mol Sci 16:19419-19432. 10.3390/ijms160819419

Li Z, Xue TQ, Yang C, Wang YL, Zhu XL, and Ni CF. 2018. EGFL7 promotes hepatocellular carcinoma cell proliferation and inhibits cell apoptosis through increasing CKS2

Peer) reviewing PDF | (2019:07:39075:2:0:NEW 5 Oct 2019) 
545

546

547

548

549

550

551

552

553

554

555

556

557

558

559

560

561

562

563

564

565

566

567

568

569

570

571

572

573

574

575

576

577

578

579

580

581

582

583

584

585

586

587

588

589

590

591

592

593

594

595

expression by activating Wnt/beta-catenin signaling. J Cell Biochem 119:10327-10337. $10.1002 / j c b .27375$

Liao X, Liu X, Yang C, Wang X, Yu T, Han C, Huang K, Zhu G, Su H, Qin W, Huang R, Yu L, Deng J, Zeng X, Ye X, and Peng T. 2018. Distinct Diagnostic and Prognostic Values of Minichromosome Maintenance Gene Expression in Patients with Hepatocellular Carcinoma. J Cancer 9:2357-2373. 10.7150/jca.25221

Nan YL, Hu YL, Liu ZK, Duan FF, Xu Y, Li S, Li T, Chen DF, and Zeng XY. 2016. Relationships between cell cycle pathway gene polymorphisms and risk of hepatocellular carcinoma. World J Gastroenterol 22:5558-5567. 10.3748/wjg.v22.i24.5558

Nie H, Li J, Yang XM, Cao QZ, Feng MX, Xue F, Wei L, Qin W, Gu J, Xia Q, and Zhang ZG. 2015. Mineralocorticoid receptor suppresses cancer progression and the Warburg effect by modulating the miR-338-3p-PKLR axis in hepatocellular carcinoma. Hepatology 62:1145-1159. 10.1002/hep.27940

Park K, and Kim KB. 2013. miRTar Hunter: a prediction system for identifying human microRNA target sites. Mol Cells 35:195-201. 10.1007/s10059-013-2165-4

Qu K, Wang Z, Fan H, Li J, Liu J, Li P, Liang Z, An H, Jiang Y, Lin Q, Dong X, Liu P, and Liu C. 2017. MCM7 promotes cancer progression through cyclin D1-dependent signaling and serves as a prognostic marker for patients with hepatocellular carcinoma. Cell Death Dis 8:e2603. 10.1038/cddis.2016.352

Ren W, Guan W, Zhang J, Wang F, and Xu G. 2019. Pyridoxine 5'-phosphate oxidase is correlated with human breast invasive ductal carcinoma development. Aging (Albany NY) 11:2151-2176. 10.18632/aging.101908

Shannon P, Markiel A, Ozier O, Baliga NS, Wang JT, Ramage D, Amin N, Schwikowski B, and Ideker T. 2003. Cytoscape: a software environment for integrated models of biomolecular interaction networks. Genome Res 13:2498-2504. 10.1101/gr.1239303

Shen DY, Fang ZX, You P, Liu PG, Wang F, Huang CL, Yao XB, Chen ZX, and Zhang ZY. 2010. Clinical significance and expression of cyclin kinase subunits 1 and 2 in hepatocellular carcinoma. Liver Int 30:119-125. 10.1111/j.1478-3231.2009.02106.x

Singal AG, and El-Serag HB. 2015. Hepatocellular Carcinoma From Epidemiology to Prevention: Translating Knowledge into Practice. Clin Gastroenterol Hepatol 13:21402151. 10.1016/j.cgh.2015.08.014

Su J, Wang Q, Liu Y, and Zhong M. 2014. miR-217 inhibits invasion of hepatocellular carcinoma cells through direct suppression of E2F3. Mol Cell Biochem 392:289-296. 10.1007/s11010-014-2039-x

Sudo T, Utsunomiya T, Mimori K, Nagahara H, Ogawa K, Inoue H, Wakiyama S, Fujita H, Shirouzu K, and Mori M. 2005. Clinicopathological significance of EZH2 mRNA expression in patients with hepatocellular carcinoma. Br J Cancer 92:1754-1758. 10.1038/sj.bjc.6602531

Sun Q, Li J, Jin B, Wang T, and Gu J. 2019a. Evaluation of miR-331-3p and miR-23b-3p as serum biomarkers for hepatitis c virus-related hepatocellular carcinoma at early stage. Clin Res Hepatol Gastroenterol. 10.1016/j.clinre.2019.03.011

Sun S, Li W, Ma X, and Luan H. 2019b. Long Noncoding RNA LINC00265 Promotes Glycolysis and Lactate Production of Colorectal Cancer through Regulating of miR-216b5p/TRIM44 Axis. Digestion:1-10. 10.1159/000500195

Sun X, Jin Z, Song X, Wang J, Li Y, Qian X, zhang Y, and Yin Y. 2015. Evaluation of KIF23 variant 1 expression and relevance as a novel prognostic factor in patients with hepatocellular carcinoma. BMC Cancer 15:961. 10.1186/s12885-015-1987-1

Tian YW, Shen Q, Jiang QF, Wang YX, Li K, and Xue HZ. 2017. Decreased levels of miR-34a and miR-217 act as predictor biomarkers of aggressive progression and poor prognosis in hepatocellular carcinoma. Minerva Med 108:108-113. 10.23736/S00264806.16.04616-4

Peer) reviewing PDF | (2019:07:39075:2:0:NEW 5 Oct 2019) 
596

597

598

599

600

601

602

603

604

605

606

607

608

609

610

611

612

613

614

615

616

617

618

619

620

621

622

623

624

625

626

627

628

629

630

631

632

633

634

635

636

637

638

639

640

641

642

643

644

645

Wang L, Su K, Wu H, Li J, and Song D. 2019. LncRNA SNHG3 regulates laryngeal carcinoma proliferation and migration by modulating the miR-384/WEE1 axis. Life Sci:116597. 10.1016/j.Ifs.2019.116597

Wen DY, Huang JC, Wang JY, Pan WY, Zeng JH, Pang YY, and Yang H. 2018. Potential clinical value and putative biological function of miR-122-5p in hepatocellular carcinoma: A comprehensive study using microarray and RNA sequencing data. Oncol Lett 16:6918-6929. 10.3892/ol.2018.9523

Wong N, and Wang X. 2015. miRDB: an online resource for microRNA target prediction and functional annotations. Nucleic Acids Res 43:D146-152. 10.1093/nar/gku1104

Xie ZC, Huang JC, Zhang LJ, Gan BL, Wen DY, Chen G, Li SH, and Yan HB. 2018. Exploration of the diagnostic value and molecular mechanism of miR1 in prostate cancer: A study based on metaanalyses and bioinformatics. Mol Med Rep 18:5630-5646. 10.3892/mmr.2018.9598

Xu H, Zhao L, Fang Q, Sun J, Zhang S, Zhan C, Liu S, and Zhang Y. 2014. MiR-338-3p inhibits hepatocarcinoma cells and sensitizes these cells to sorafenib by targeting hypoxiainduced factor 1alpha. PLoS One 9:e115565. 10.1371/journal.pone.0115565

Xu M, Chen X, Lin K, Zeng K, Liu X, Pan B, Xu X, Xu T, Hu X, Sun L, He B, Pan Y, Sun H, and Wang S. 2018. The long noncoding RNA SNHG1 regulates colorectal cancer cell growth through interactions with EZH2 and miR-154-5p. Mol Cancer 17:141. 10.1186/s12943018-0894-X

Yang T, He X, Chen A, Tan K, and Du X. 2018a. LncRNA HOTAIR contributes to the malignancy of hepatocellular carcinoma by enhancing epithelial-mesenchymal transition via sponging miR-23b-3p from ZEB1. Gene 670:114-122. 10.1016/j.gene.2018.05.061

Yang XY, Wu B, Ma SL, Yin L, Wu MC, and Li AJ. 2018b. Decreased Expression of ZWINT is Associated With Poor Prognosis in Patients With HCC After Surgery. Technol Cancer Res Treat 17:1533033818794190. 10.1177/1533033818794190

Yin H, Hu M, Zhang R, Shen Z, Flatow L, and You M. 2012. MicroRNA-217 promotes ethanolinduced fat accumulation in hepatocytes by down-regulating SIRT1. J Biol Chem 287:9817-9826. 10.1074/jbc.M111.333534

Ying H, Xu Z, Chen M, Zhou S, Liang X, and Cai X. 2018. Overexpression of Zwint predicts poor prognosis and promotes the proliferation of hepatocellular carcinoma by regulating cell-cycle-related proteins. Onco Targets Ther 11:689-702. 10.2147/OTT.S152138

You Y, Tan J, Gong Y, Dai H, Chen H, Xu X, Yang A, Zhang Y, and Bie P. 2017. MicroRNA216b-5p Functions as a Tumor-suppressive RNA by Targeting TPT1 in Pancreatic Cancer Cells. J Cancer 8:2854-2865. 10.7150/jca.18931

Zeng JF, Zeng ZL, Zhang K, Zhao Y, Liu YM, Chen JJ, Tong H, Wei DH, Jiang ZS, and Wang Z. 2018. miR-23b-3p and miR-125b-5p downregulate apo(a) expression by targeting Ets1 in HepG2 cells. Cell Biol Int 42:313-323. 10.1002/cbin.10896

Zhang H, Zhou D, Ying M, Chen M, Chen P, Chen Z, and Zhang F. 2016a. Expression of Long Non-Coding RNA (IncRNA) Small Nucleolar RNA Host Gene 1 (SNHG1) Exacerbates Hepatocellular Carcinoma Through Suppressing miR-195. Med Sci Monit 22:4820-4829. $10.12659 / \mathrm{msm} .898574$

Zhang M, Li M, Li N, Zhang Z, Liu N, Han X, Liu Q, and Liao C. 2017. miR-217 suppresses proliferation, migration, and invasion promoting apoptosis via targeting MTDH in hepatocellular carcinoma. Oncol Rep 37:1772-1778. 10.3892/or.2017.5401

Zhang M, Wang W, Li T, Yu X, Zhu Y, Ding F, Li D, and Yang T. 2016b. Long noncoding RNA SNHG1 predicts a poor prognosis and promotes hepatocellular carcinoma tumorigenesis. Biomed Pharmacother 80:73-79. 10.1016/j.biopha.2016.02.036

Zhang PF, Wang F, Wu J, Wu Y, Huang W, Liu D, Huang XY, Zhang XM, and Ke AW. 2019a. LncRNA SNHG3 induces EMT and sorafenib resistance by modulating the miR-

Peerj reviewing PDF | (2019:07:39075:2:0:NEW 5 Oct 2019) 
646

647

648

649

650

651

652

653

654

655

656

657

658

659

660

661

662
128/CD151 pathway in hepatocellular carcinoma. J Cell Physiol 234:2788-2794. 10.1002/jcp.27095

Zhang T, Cao C, Wu D, and Liu L. 2016c. SNHG3 correlates with malignant status and poor prognosis in hepatocellular carcinoma. Tumour Biol 37:2379-2385. 10.1007/s13277-0154052-4

Zhang T, Liu W, Zeng XC, Jiang N, Fu BS, Guo Y, Yi HM, Li H, Zhang Q, Chen WJ, and Chen GH. 2016d. Down-regulation of microRNA-338-3p promoted angiogenesis in hepatocellular carcinoma. Biomed Pharmacother 84:583-591. 10.1016/j.biopha.2016.09.056

Zhang Z, Li J, He T, Ouyang Y, Huang Y, Liu Q, Wang P, and Ding J. 2019b. The competitive endogenous RNA regulatory network reveals potential prognostic biomarkers for overall survival in hepatocellular carcinoma. Cancer Sci. 10.1111/cas.14138

Zheng S, Jiang F, Ge D, Tang J, Chen H, Yang J, Yao Y, Yan J, Qiu J, Yin Z, Ni Y, Zhao L, Chen X, Li H, and Yang L. 2019. LncRNA SNHG3/miRNA-151a-3p/RAB22A axis regulates invasion and migration of osteosarcoma. Biomed Pharmacother 112:108695. 10.1016/j.biopha.2019.108695 
Figure 1

Identification of DElncRNAs and DEGs

(A) Volcano plots of the gene expression profile data. The blue dots represent downregulated genes and the orange dots represent up-regulated genes (B) Heat map of IncRNAs in HCC samples compared with normal samples.

A

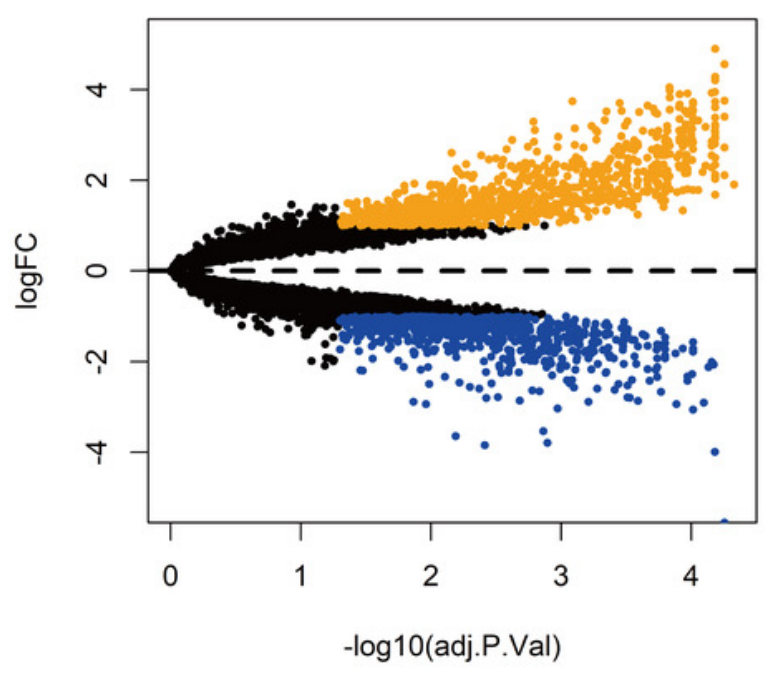

B

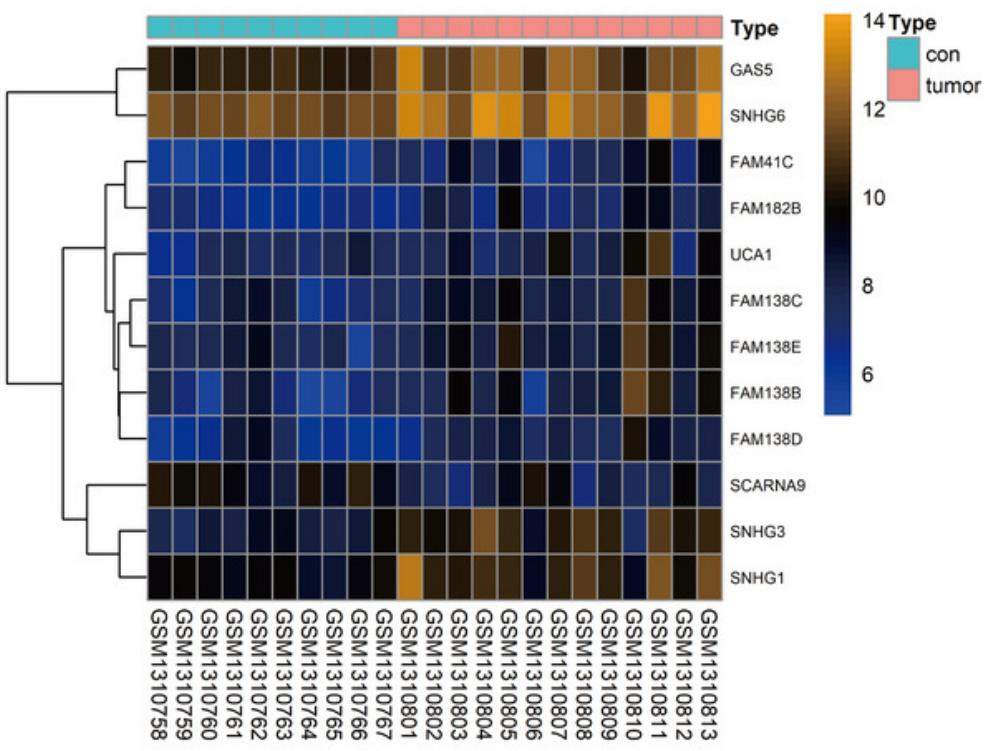


Figure 2

IncRNA-associated ceRNA network.

Red nodes represent the 11 DEIncRNAs. Green nodes represent the 32 predictive miRNAs. Blue nodes represent the 126 predictive mRNAs. 


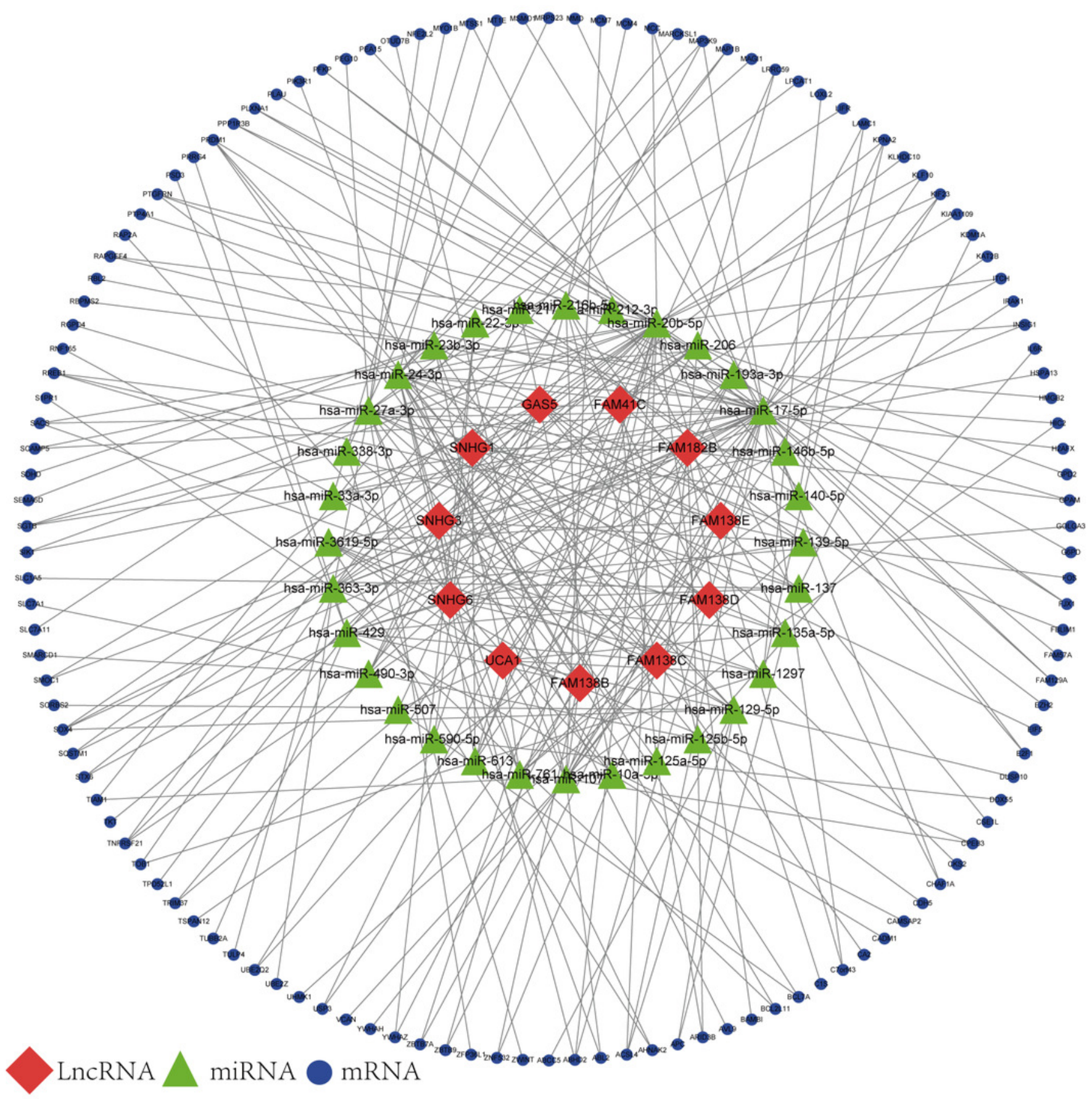


Figure 3

GO and KEGG analysis of DEGs.

\section{(A) Biological processes, (B) Cell components, (C) Molecular functions of GO} analysis. (D) KEGG analysis

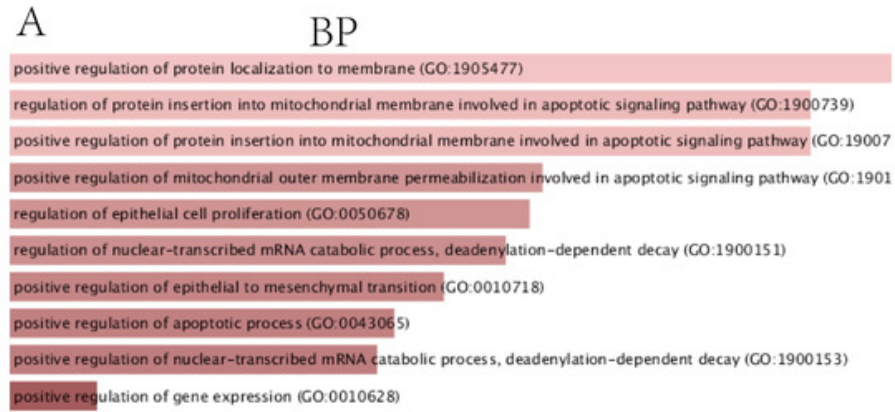

C $\mathrm{MF}$

trans cription regulatory region DNA binding (CO. 0044212)

core promoter sequence-specific DNA binding (CO:0001 046)

mRNA 3'-UTR AU-rich region binding (CO.0035925)

regulatory region DNA bindirg (CO:0000975)

RNA polymerase II activating transcription factor binding (C0:0001102)

core promoter binding (C):0001047)

cadherin binding (G0.045296)

L-amino acid transmembrane transporter activity (CO:0015179)

insulin-like growth factor receptor binding (c0:0005159)

amino acid transmembrane transporter activity (Co:0015171)
B CC

chromatin (Co:0000785) nuclear chromosome part (CO:0044454)

nuclear chromatin (CO. 0000790$)$

apical dendrite (CO.0097440)

microtubule organizing center (C0.0005815)

cyoplasmic vesicle membrane (C0:0030659)

ESC/EZ) complex (C0:0035098)

lipid droplet (C0:0005811)

chromosome, telomeric region (C0:0000781)

centrosome (CO:0005813)

D KEGG

Cell cycle

MicroRNAs in cancer

Central carbon metabolism in cancer

Pentose phosphate pathway

P13K-Akt signaling pathway

Fluid shear stress and atherosclerosis

Colorectal cancer

Non-alcoholic fatty lifer disease (NAFLD)

Small cell lung carcer

Cellular serescence 
Figure 4

PPI network of DEGs and hub genes.

(A) PPI network of DEGs. Blue nodes represent the interaction among DEGs. Only the 90 DEGs that interacting with other ones were demonstrated in the network. (B) 10 Hub genes identified from the PPI network. From the red nodes to yellow ones, the connection degree of each molecule with others gradually decreases. 


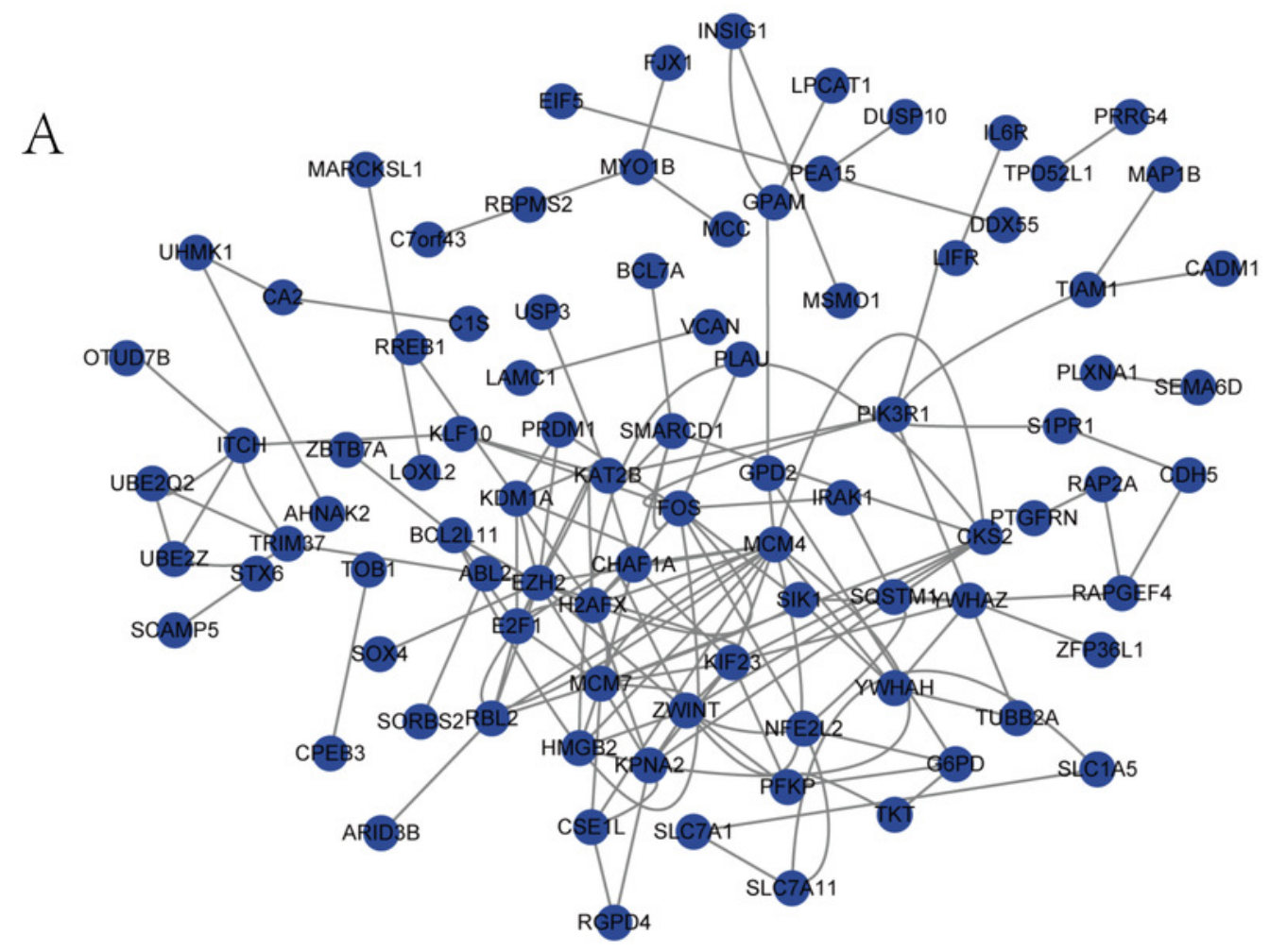

B

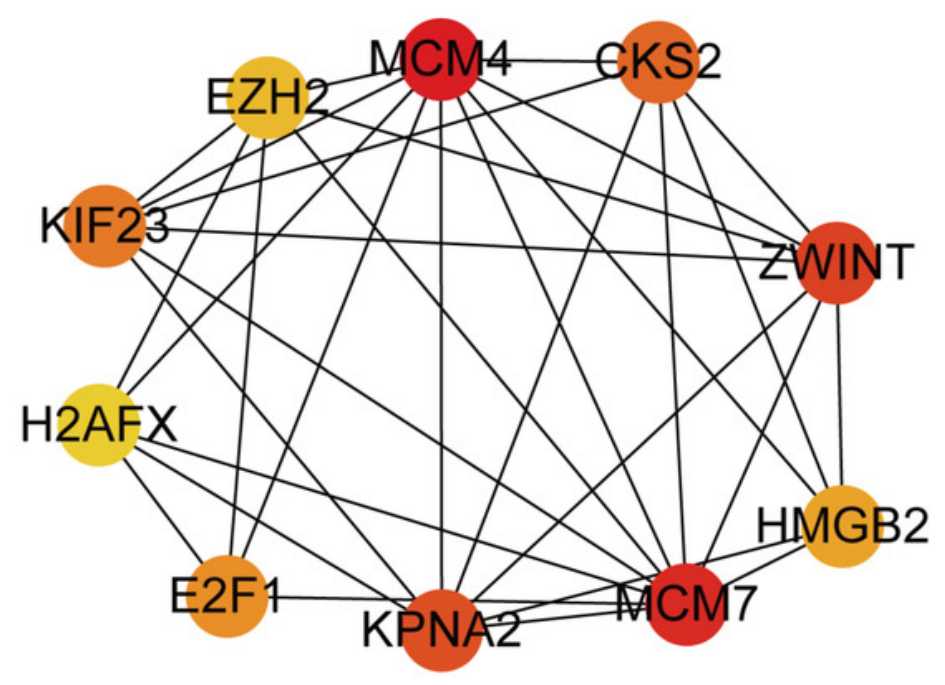


Figure 5

Overall survival analysis of the hub genes.

(A) MCM4. (B) MCM7. (C) ZWINT. (D) KPNA2. (E) CKS2. (F) KIF23. (G) E2F1. (H) HMGB2. (I) EZH2. (J) H2AFX. The overall survival of patients with highly expressed hub genes was significantly shorter than those with low expression. 

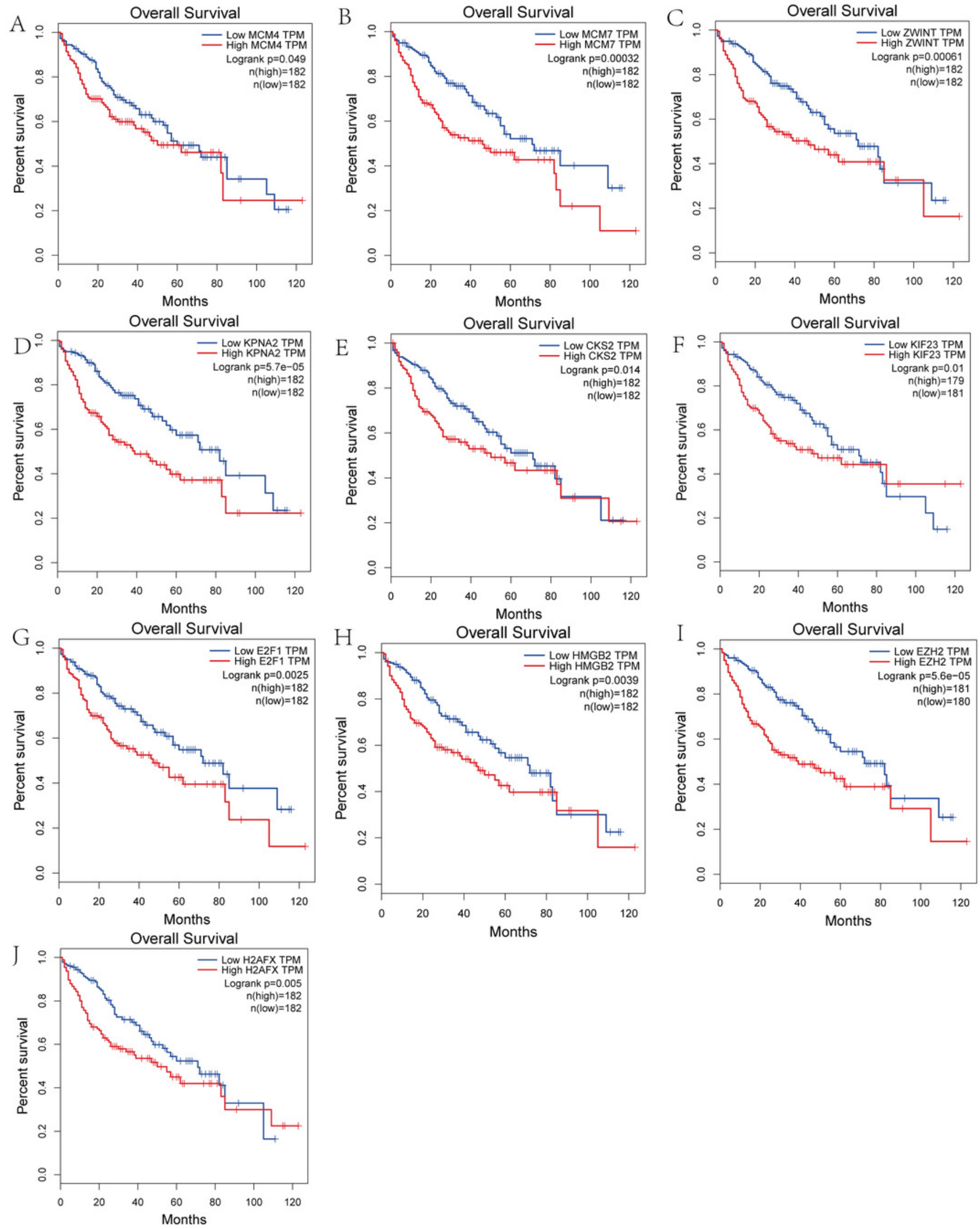
Figure 6

Overall survival analysis of the DEIncRNAs.

(A) FAM182B. (B) SNHG1. (C) SNHG3. (D) SNHG6. The overall survival of patients with highly expressed DEInCRNAs was significantly shorter than those with low expression. 

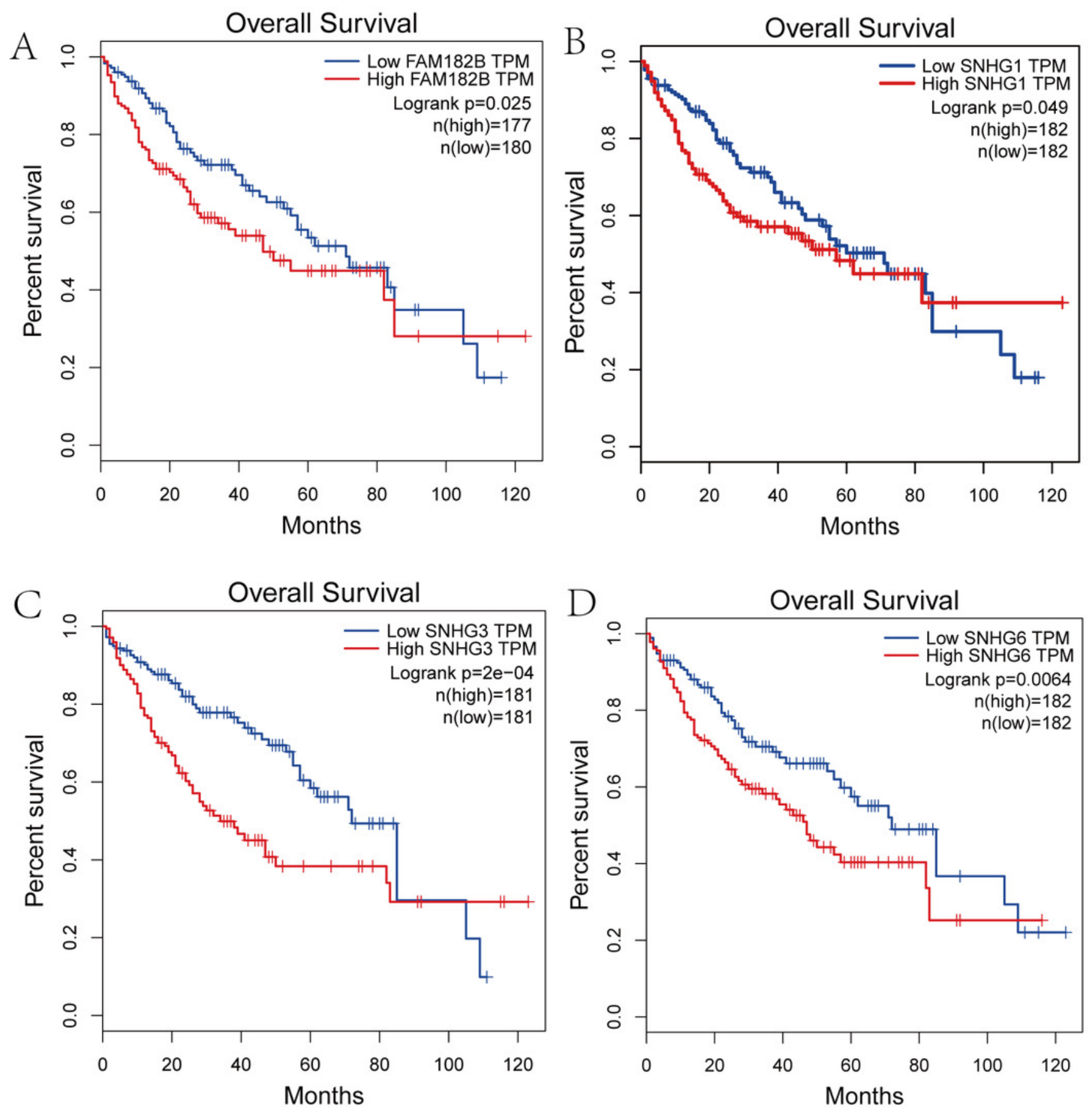
Figure 7

Validation of IncRNA expression in UALCAN database.

(A)FAM182B. (B) SNHG1. (C) SNHG3. (D) SNHG6. Based on UALCAN, all DEInCRNAS were significantly overexpressed in HCC tissues compared with the normal ones.

A Expression of FAM182B in LIHC based on Sample types

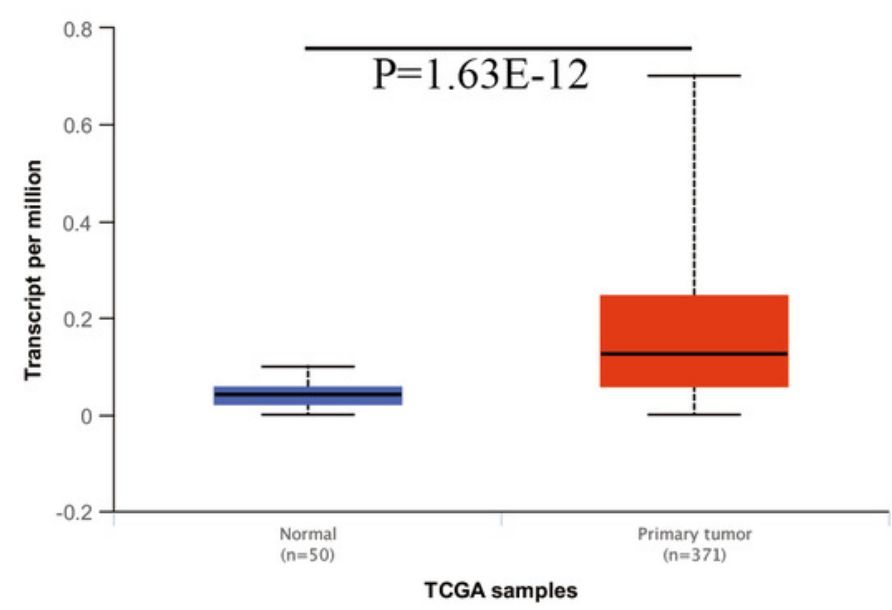

C Expression of SNHG3 in LIHC based on Sample types

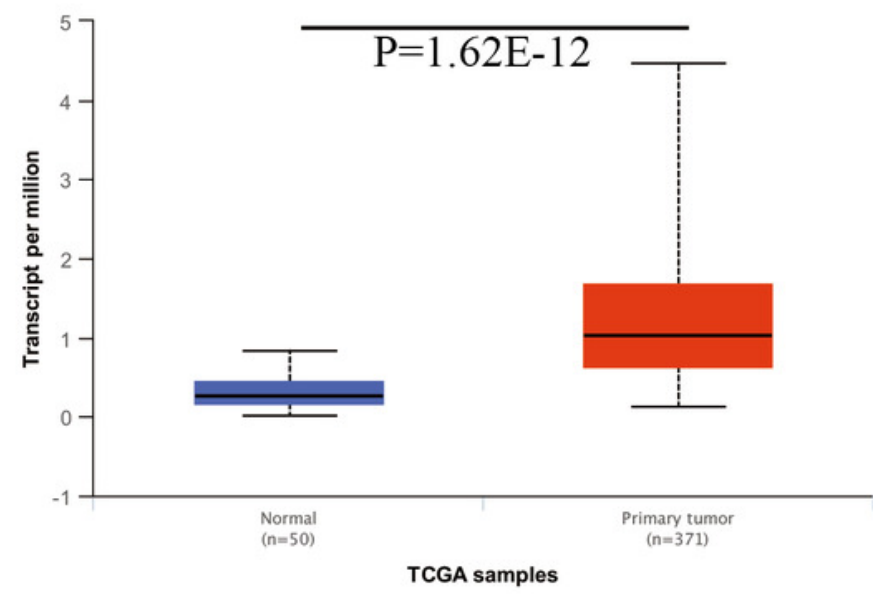

B Expression of SNHG1 in LIHC based on Sample types

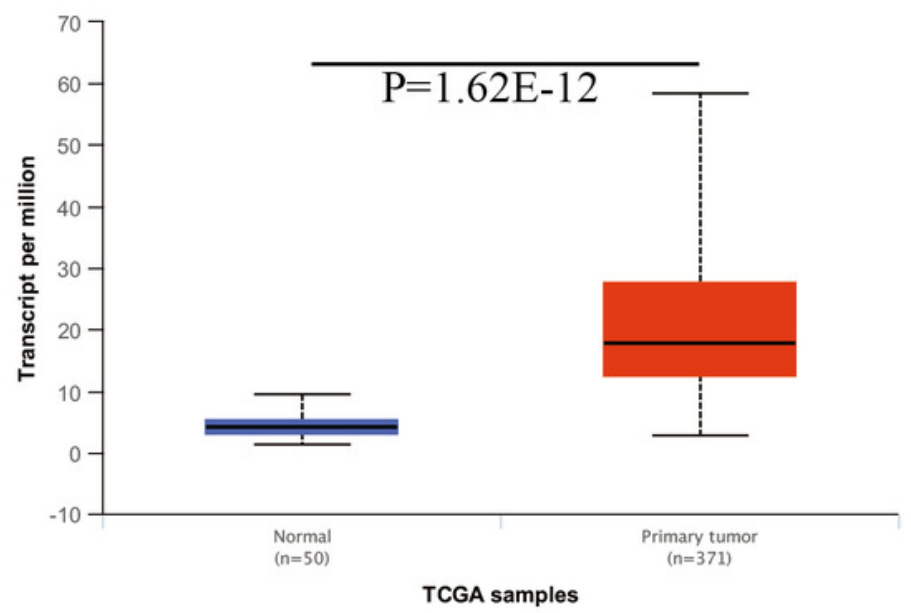

D Expression of SNHG6 in LIHC based on Sample types

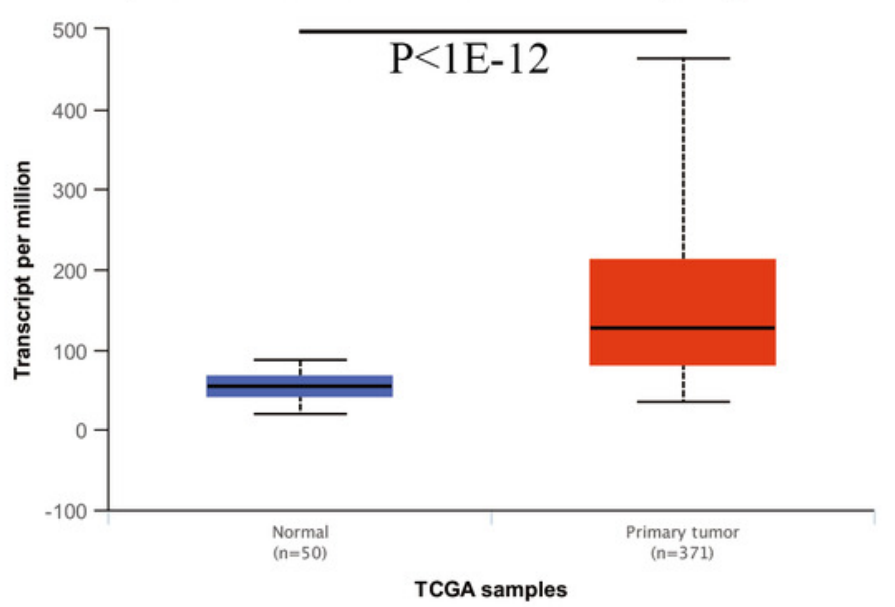


Figure 8

Validation of hub genes expression in UALCAN database.

(A) MCM4. (B) MCM7. (C) ZWINT. (D) KPNA2. (E) CKS2. (F) KIF23. (G) E2F1. (H) HMGB2. (I) EZH2. (J) H2AFX. Based on UALCAN, all hub genes were significantly overexpressed in HCC tissues compared with the normal ones. 
A Expression of MCM4 in UHC based on Sample types

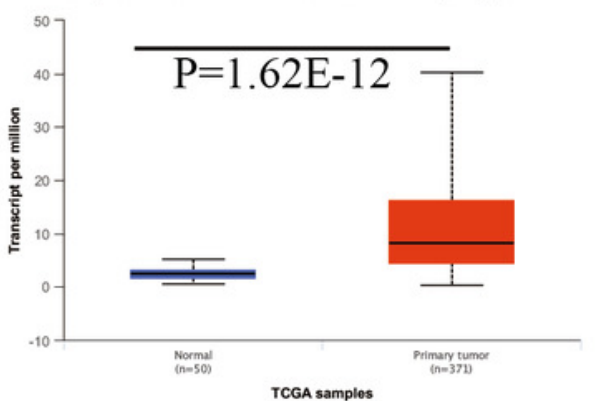

D Expression of KPNAz in UHC based on Sample types

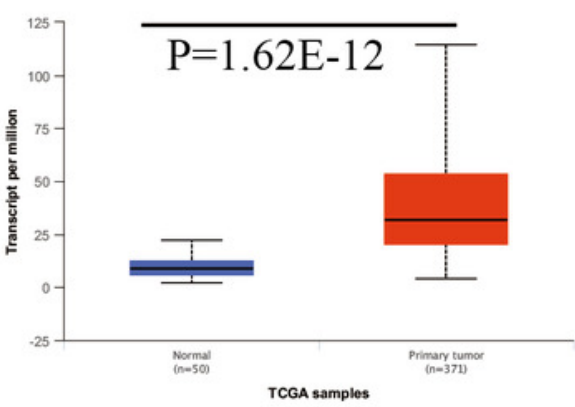

G Expression of E2F1 in LHC based on Sample types

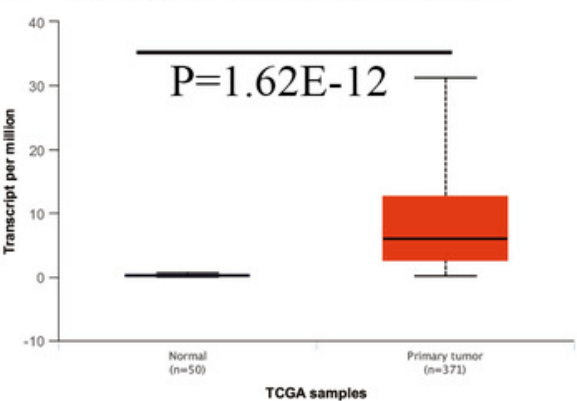

J Expression of HZAFX in LHC based on Sample types

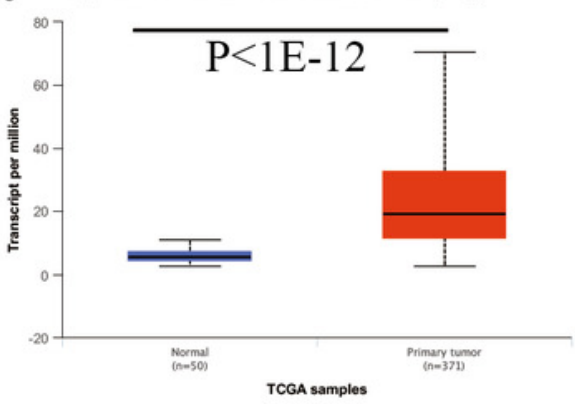

B Expression of MCM7 in LHC based on Sample types

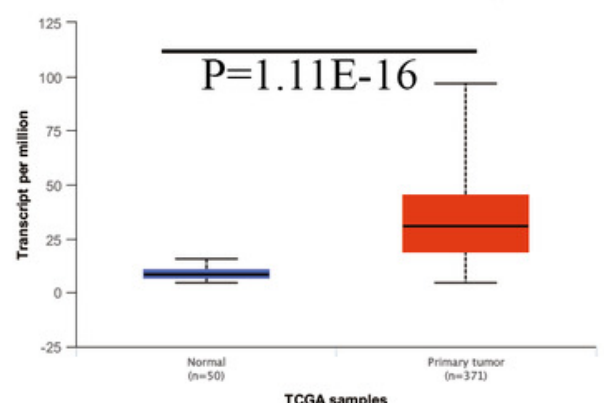

E

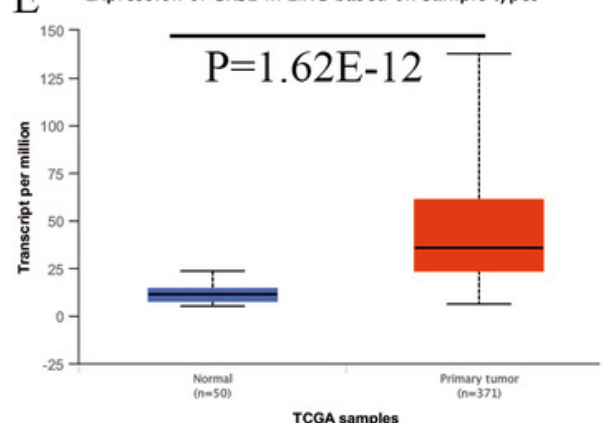

H Expression of HMGB2 in LHHC based on Sample types

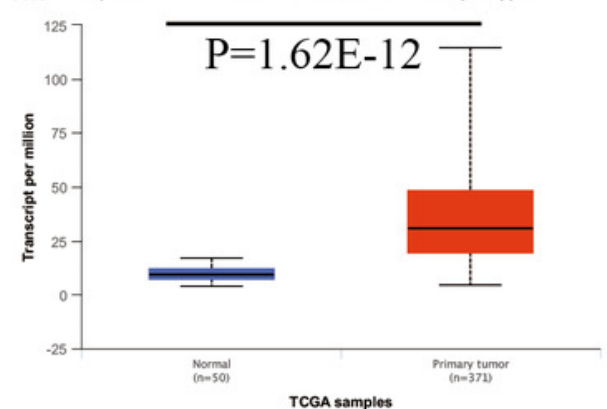

C Expression of ZWINT in LHC based on Sample types

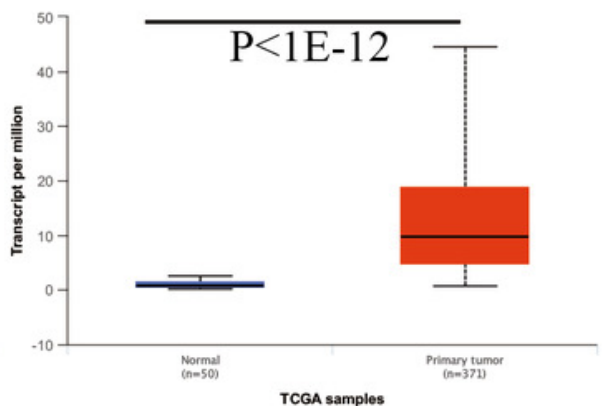

F Expression of KIF23 in LHC based on Sample types

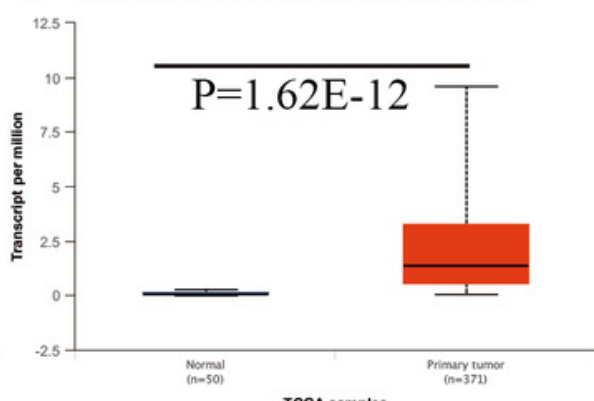

I Expression of EZH2 in $\mathrm{LHC}$ based on Sample types

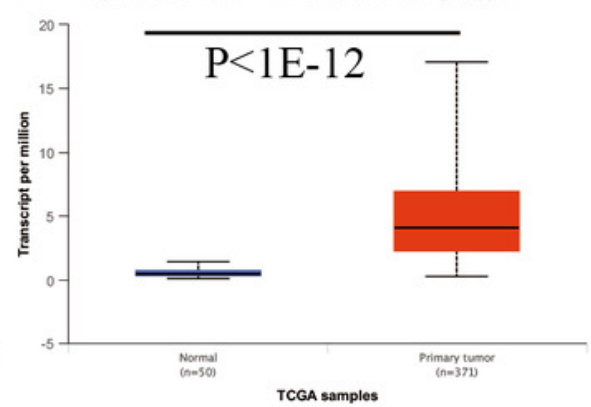




\section{Figure 9}

Validation of hepatic expression of hub genes in The Human Protein Atlas database.

Expression of (A) MCM4, (C) KIF23, (E) MCM7, (G) E2F1, (I) ZWINT, (K) HMGB2, (M) KPNA2, (O) EZH2, (Q) CKS2, (S) H2AFX in non-tumor tissues. Expression of (B) MCM4, (D) KIF23, (F) MCM7, (H) E2F1, (J) ZWINT, (L) HMGB2, (N) KPNA2, (P) EZH2, (R) CKS2, (T) H2AFX in tumor tissues. Proteins encoded by MCM4, MCM7, ZWINT, CKS2, E2FI, HMGB2 and EZH2 were expressed higher in tumor than in non-tumor tissues. KPNA2 were expressed moderately in both groups. KIF23 and H2AFX were highly expressed in both tumor and non-tumor tissues. 

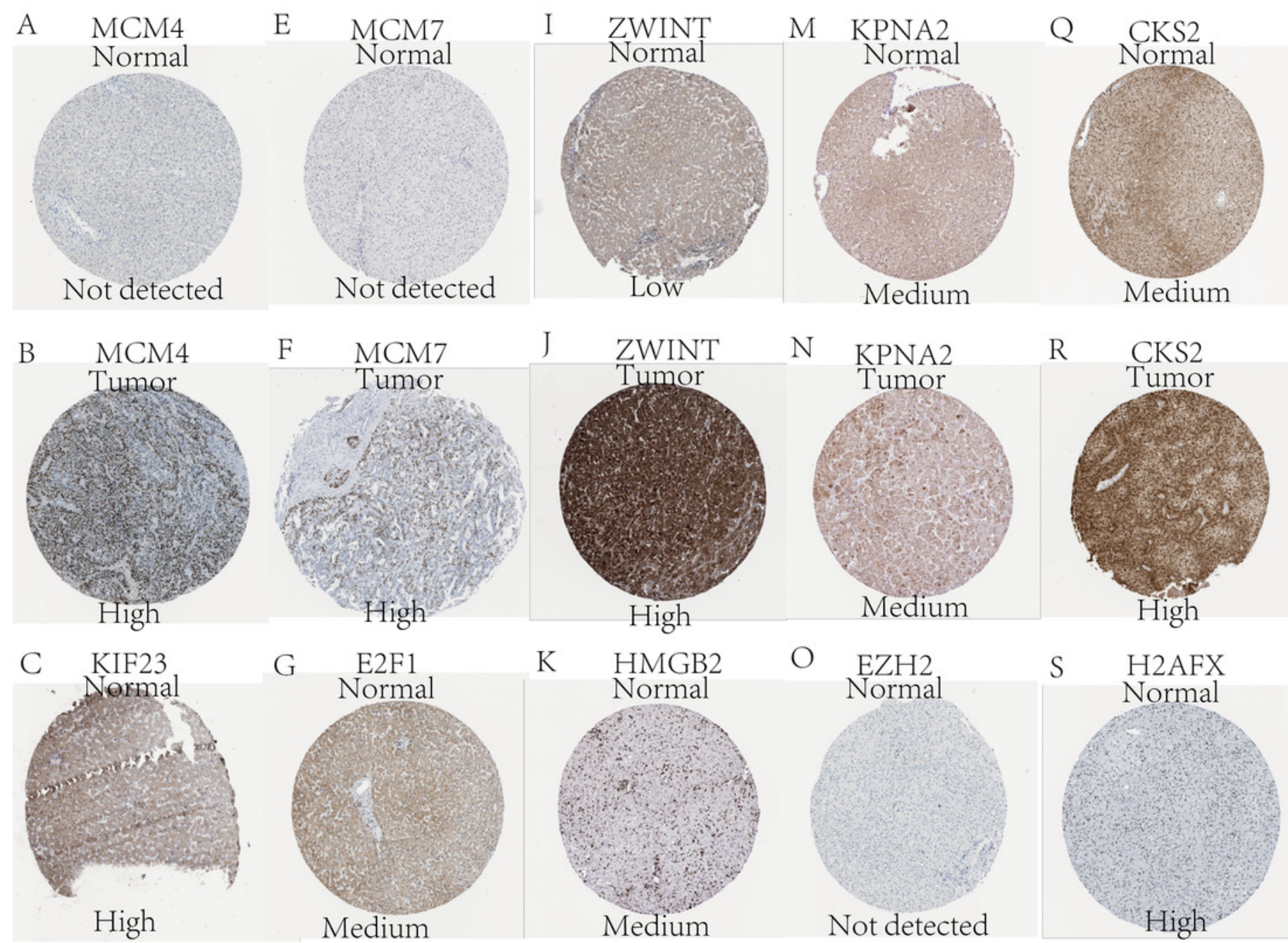

O $\underset{\text { Normal }}{\mathrm{EZH} 2}$

S H2AFX
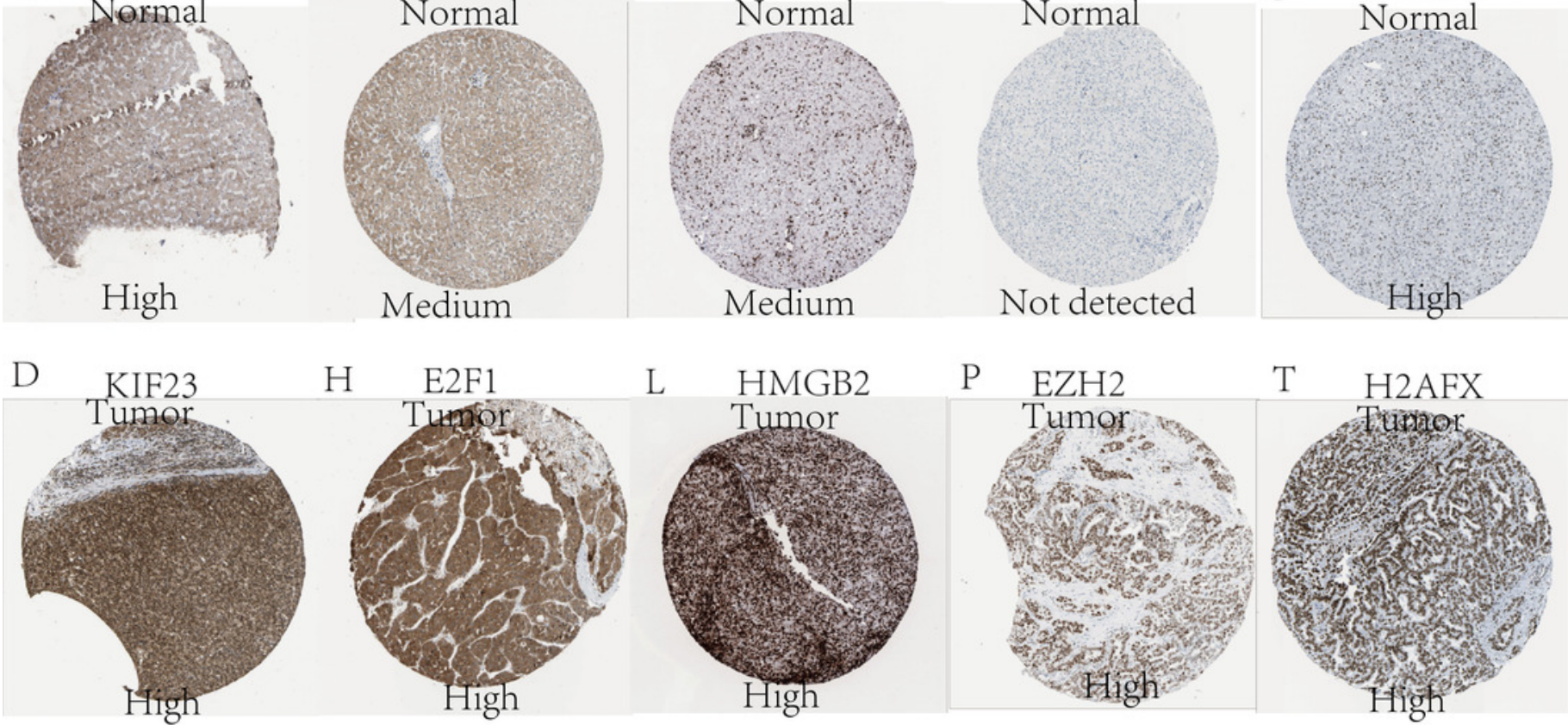


\section{Figure 10}

The IncRNA-miRNA-mRNA ceRNA network constructed from hub genes and DEIncRNAs.

Red nodes represent InCRNAs. Green nodes represent miRNAs. Blue nodes represent hub genes. 10 IncRNA-miRNA-mRNA pathways were reconstructed here. 


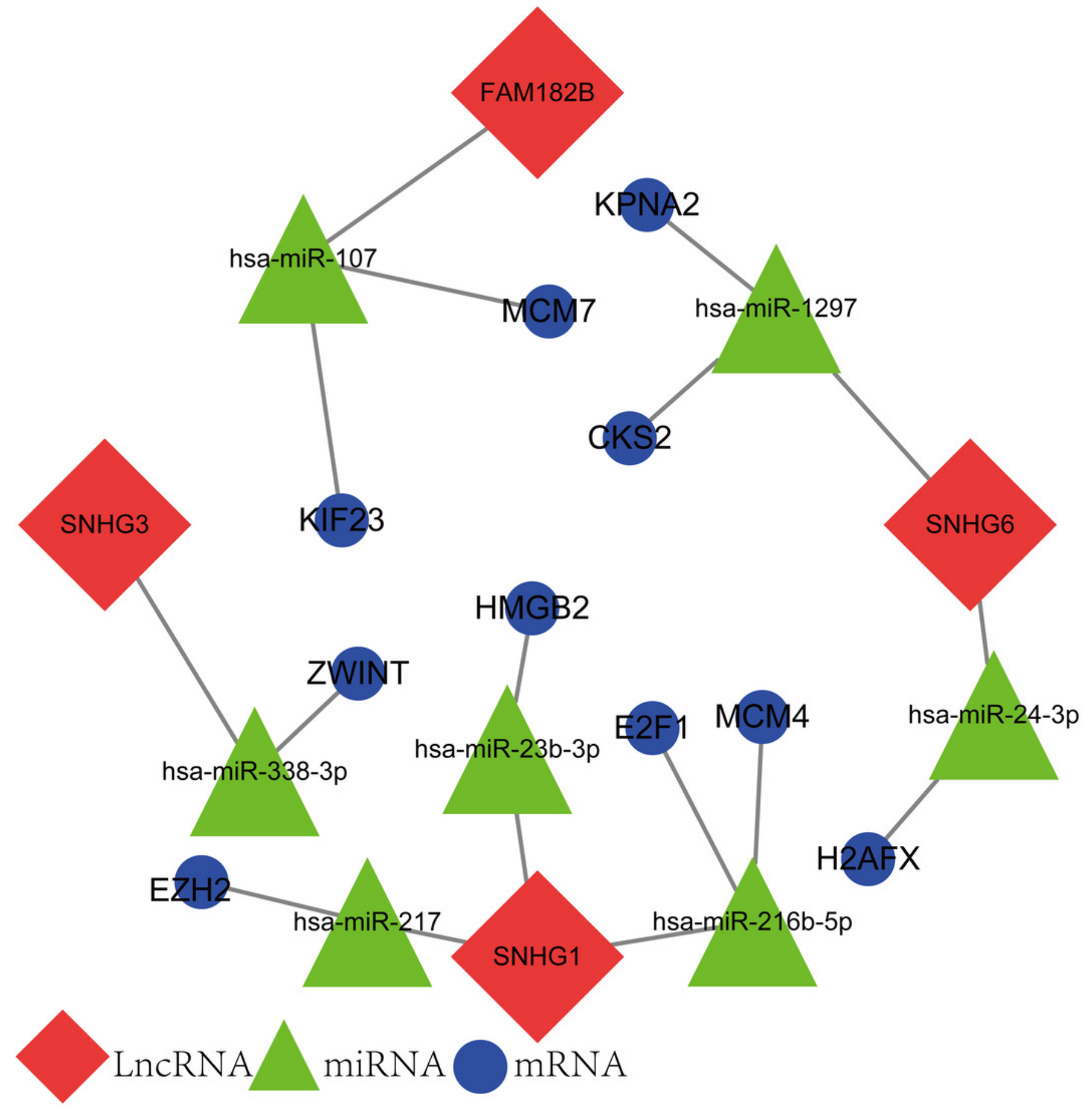




\section{Figure 11}

Co-expression analysis of DEIncRNAs from ceRNA network with related hub genes in HCC patients.

(A) InCRNA FAM182B with KIF23. (B) InCRNA FAM182B with MCM7. (C) InCRNA SNHG1 with

E2F1. (D) InCRNA SNHG1 with EZH2. (E) InCRNA SNHG1 with HMGB2. (F) InCRNA SNHG1 with MCM4. (G) InCRNA SNHG3 with ZWINT. (H) InCRNA SNHG6 with CKS2. (I) InCRNA SNHG6 with H2AFX. (J) InCRNA SNHG6 with KPNA2. SNHG1 and SNHG3 were strongly correlatted with their hub genes. FAM182B and SNHG6 showed moderate correlation with corresponding hub genes. 

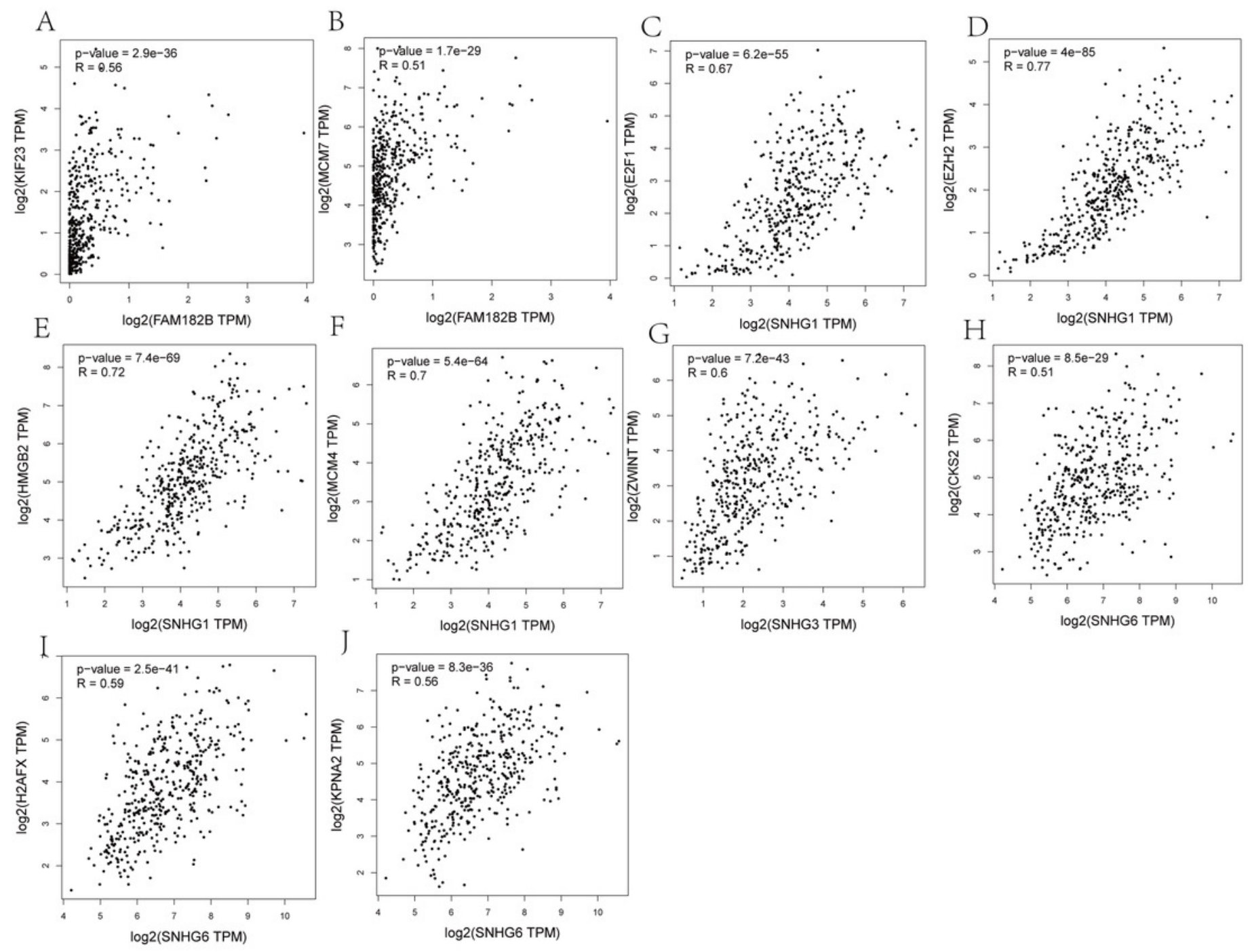
Table $\mathbf{1}$ (on next page)

Reconstruction of the IncRNA-associated ceRNA networks.

Reconstruction of the IncRNA-associated ceRNA networks. 
1 Table 1 Reconstruction of the lncRNA-associated ceRNA network

2

\begin{tabular}{ccc}
\hline IncRNA & miRNA & mRNA \\
\hline SNHG1 & has-miR-216b-5P & E2F1 \\
& hsa-miR-217 & MCM4 \\
& has-miR-23b-3P & EZH2 \\
SNHG6 & has-miR-1297 & HMGB2 \\
& & KPNA2 \\
FAM182B & has-miR-24-3P & CKS2 \\
& hsa-miR-107 & H2AFX \\
SNHG3 & has-miR-338-3P & MCM7 \\
\hline
\end{tabular}

